\title{
ACCURATE ARRANGEMENTS
}

\author{
PAUL MÜCKSCH AND GERHARD RÖHRLE
}

ABSTRACT. Let $\mathscr{A}$ be a Coxeter arrangement of rank $\ell$. In 1987 Orlik, Solomon and Terao conjectured that for every $1 \leq d \leq \ell$, the first $d$ exponents of $\mathscr{A}$ - when listed in increasing order are realized as the exponents of a free restriction of $\mathscr{A}$ to some intersection of reflecting hyperplanes of $\mathscr{A}$ of dimension $d$.

This conjecture does follow from rather extensive case-by-case studies by Orlik and Terao from 1992 and 1993, where they show that all restrictions of Coxeter arrangements are free.

We call a general free arrangement with this natural property involving their free restrictions accurate. In this paper we initialize their systematic study.

Our principal result shows that MAT-free arrangements, a notion recently introduced by Cuntz and Mücksch, are accurate.

This theorem in turn directly implies this special property for all ideal subarrangements of Weyl arrangements. In particular, this gives a new, simpler and uniform proof of the aforementioned conjecture of Orlik, Solomon and Terao for Weyl arrangements which is free of any case-by-case considerations.

Another application of a slightly more general formulation of our main theorem shows that extended Catalan arrangements, extended Shi arrangements, and ideal-Shi arrangements share this property as well.

We also study arrangements that satisfy a slightly weaker condition, called almost accurate arrangements, where we simply disregard the ordering of the exponents involved. This property in turn is implied by many well established concepts of freeness such as supersolvability and divisional freeness.

\section{Contents}

1. Introduction

2. Preliminaries

2010 Mathematics Subject Classification. Primary: 20F55; Secondary: 51F15, $52 \mathrm{C} 35,32 \mathrm{~S} 22$.

Key words and phrases. Free arrangements, Coxeter arrangements, Weyl arrangements, Ideal arrangements, MAT-free arrangements, accurate arrangements, extended Catalan arrangements, extended Shi arrangements, ideal-Shi arrangements. 
2.1. Hyperplane arrangements 6

2.2. Free arrangements $\quad 7$

2.3. Topological incarnation of the module of $\mathscr{A}$-derivations 9

2.4. MAT-free arrangements 10

3. Proof of Theorem $1.2 \quad 12$

3.1. Basis derivations across MAT-steps 12

3.2. Restrictions of MAT-free arrangements 14

3.3. A generalization of Theorem $1.2 \quad 16$

4. Applications 18

4.1. Weyl and Ideal arrangements 18

4.2. Ideal-Shi and extended Catalan arrangements 20

5. Complements and Examples 23

5.1. Almost accurate arrangements 23

5.2. Graphic arrangements 25

5.3. Complex reflection arrangements and their restrictions 26

References $\quad 30$

\section{INTRODUCTION}

The Coxeter arrangement $\mathscr{A}(W)$ of a Coxeter group $W$ is the hyperplane arrangement in the reflection representation of $W$ which consists of the reflecting hyperplanes associated with the reflections in $W$. Coxeter arrangements are pivotal to the theory of hyperplane arrangements. In the late 1970s Arnold [Arn76], [Arn79] and Saito [Sai75], [Sai80] showed independently that every Coxeter arrangement is free and that its exponents are given by the exponents of the underlying Coxeter group.

In [OS83], Orlik and Solomon computed the characteristic polynomial of every restriction $\mathscr{A}(W)^{X}$ of each Coxeter arrangement $\mathscr{A}(W)$ to some intersection $X$ of reflecting hyperplanes of $\mathscr{A}(W)$ in long and intricate computations. They showed that each such factors over $\mathbb{Z}$. Moreover, they observed that for each $1 \leq d \leq \ell$, where $\ell$ is the dimension of the reflection representation of $W$, there exists such a restriction $\mathscr{A}(W)^{X}$ such that the roots of the characteristic polynomial of $\mathscr{A}(W)^{X}$ are the first $d$ exponents of $\mathscr{A}(W)$ when ordered by size.

In view of this fact, owing to [OST87, Thm. 1.12] (see Theorem 1.5), and Terao's Factorization Theorem for free arrangements [Ter81] (cf. [OT92, Thm. 4.137]), Orlik, Solomon and Terao conjectured in [OST87, Conj. 1.14] that an even stronger property might hold. Namely that the first $d$ exponents of $\mathscr{A}(W)$ when ordered by size are realized 
as the exponents of some free restriction $\mathscr{A}(W)^{X}$ of $\mathscr{A}(W)$ for every $1 \leq d \leq \ell$ (Theorem 1.6), where $\ell$ is as above.

In long and intricate case-by-case studies, Orlik and Terao showed in [OT92, §6.4] and [OT93] that indeed all the restricted arrangements $\mathscr{A}(W)^{X}$ are free for a Coxeter group $W$. In case $W$ is a Weyl group, Douglass [Dou99, Cor. 6.1] gave a uniform proof of this fact by means of an elegant, conceptual Lie theoretic argument. But the latter does not provide any information on the exponents of the restrictions at hand.

From the numerical data obtained in [OS83] and the results from [OT92, §6.4] and [OT93] along with [OST87, Thm. 1.12] one can readily confirm the aforementioned conjecture [OST87, Conj. 1.14] simply by checking all instances - the only known proof of this conjecture to date. As a consequence of our results, we give a new proof of this fact in the case of Weyl arrangements which is uniform and free of any case-by-case considerations.

The goal of this paper is to initialize a systematic study of this natural property in general.

Definition 1.1. An arrangement $\mathscr{A}$ is said to be accurate if $\mathscr{A}$ is free with exponents $\exp (\mathscr{A})=\left(e_{1} \leq e_{2} \leq \ldots \leq e_{\ell}\right)$ and for every $1 \leq d \leq \ell$ there exists an intersection $X$ of hyperplanes from $\mathscr{A}$ of dimension $d$ such that $\mathscr{A}^{X}$ is free with $\exp \left(\mathscr{A}^{X}\right)=\left(e_{1} \leq e_{2} \leq \ldots \leq e_{d}\right)$.

In $\left[\mathrm{ABC}^{+} 16\right]$, Abe, Barakat, Cuntz, Hoge and Terao proved the socalled Multiple Addition Theorem (MAT) (Theorem 2.10) which is a variation of the addition part of Terao's seminal Addition-Deletion Theorem [Ter80a] ([OT92, Thm. 4.51]). Using this theorem, they went on to uniformly derive the freeness of ideal subarrangements of Weyl arrangements (Definition 4.1 and Theorem 4.2). As a special case of this result, they obtained a new uniform proof of the classical Kostant-Macdonald-Shapiro-Steinberg formula for the exponents of a Weyl group.

In [CM20], Cuntz and Mücksch introduced the notion of MATfreeness (Definition 2.14) to investigate arrangements whose freeness can be derived using an iterative application of the Multiple Addition Theorem (Theorem 2.10), similar to the class of ideal arrangements.

Our principal result asserts that MAT-freeness is sufficient for accuracy from Definition 1.1.

Theorem 1.2. MAT-free arrangements are accurate. 
Indeed, we prove a more detailed result in Theorem 3.9 as follows. Firstly, we determine which restrictions $\mathscr{A}^{X}$ of an MAT-free arrangement $\mathscr{A}$ realize the subsequences of the exponents of $\mathscr{A}$. They are the intersections of hyperplanes contained in one of the blocks of a certain partition associated to every MAT-free arrangement, a so called MAT-partition (Definition 2.14).

Secondly, we delineate bases of the derivation module of an MATfree arrangement $\mathscr{A}$ which restrict to bases of the derivation modules of the relevant restricted arrangements $\mathscr{A}^{X}$. For details see Theorem 3.9 .

The methods we use to derive our results are inspired in part by arguments employed in the proof of Theorem 2.10 from $\left[\mathrm{ABC}^{+} 16\right]$.

As ideal subarrangements of Weyl arrangements are MAT-free, by $\left[\mathrm{ABC}^{+} 16\right]$, Theorem 1.2 readily yields our next key result.

Theorem 1.3. Ideal arrangements are accurate.

The blocks of the associated MAT-partition are realized by roots of the same height in the given ideal. Hence in the case of ideal arrangements the intersections of hyperplanes realizing the particular exponents are given by antichains in the root poset consisting of roots of the same height. For further details see Section 4.1.

We record the following special case from Theorem 1.3.

Corollary 1.4. Let $\mathscr{A}=\mathscr{A}(W)$ be an irreducible Weyl arrangement with $\exp (\mathscr{A})=\left(e_{1} \leq e_{2} \leq \ldots \leq e_{\ell}\right)$ and let $H \in \mathscr{A}$ be the hyperplane corresponding to the highest root of an irreducible root system associated to $W$. Then $\mathscr{A}^{H}$ is free with $\exp \left(\mathscr{A}^{H}\right)=\left(e_{1} \leq e_{2} \leq \ldots \leq e_{\ell-1}\right)$.

The highest root of an irreducible root system associated to the Weyl group $W$ is always long (cf. [Bou68, Ch. VI, Prop. 25]) and roots of the same length are conjugate under the action of $W$ (cf. [Bou68, Ch. VI, Prop. 11]). Further, as the linear transformation mapping a root system to its dual, interchanging long and short roots, fixes the ambient Weyl arrangement (cf. [Bou68, Ch. VI, Prop. 1 ff.]), we recover the following classical result already mentioned above for the case of Weyl arrangements from Corollary 1.4.

Theorem 1.5 ([OST87, Thm. 1.12]). Let $\mathscr{A}=\mathscr{A}(W)$ be an irreducible Coxeter arrangement with $\exp (\mathscr{A})=\left(e_{1} \leq e_{2} \leq \ldots \leq e_{\ell}\right)$ and $H \in \mathscr{A}$. Then $\mathscr{A}^{H}$ is free with $\exp \left(\mathscr{A}^{H}\right)=\left(e_{1} \leq e_{2} \leq \ldots \leq e_{\ell-1}\right)$.

Because all Coxeter arrangements are MAT-free, owing to $\left[\mathrm{ABC}^{+} 16\right]$ and [CM20], we obtain our third chief result as a consequence of Theorem 1.2 . 
Theorem 1.6 ([OST87, Conj. 1.14]). Coxeter arrangements are accurate.

Remarks 1.7. (i) In $\left[\mathrm{ABC}^{+} 16\right]$, MAT-freeness is derived uniformly for the Weyl arrangement case. So our methods give a new uniform proof of Theorem 1.6 for this case which is considerably simpler than the original one indicated above, and most importantly it does not require the classification of the Weyl arrangements nor that of their restrictions.

(ii) The converse of Theorem 1.2 is false. The rank 3 supersolvable simplicial arrangement $\mathscr{A}(10,1)$ with 10 hyperplanes (cf. [Grü09]) is accurate but it is not MAT-free, by [CM20, Ex. 3.10].

(iii) In general an accurate arrangement $\mathscr{A}$ may admit a free restriction whose exponents fail to be a subset of the exponents of $\mathscr{A}$, e.g. the lattice of the reflection arrangement of the Coxeter group of type $E_{6}$ admits several such instances.

It even might be the case that an accurate arrangement admits a restriction which is not free at all. For instance, there are examples of ideal subarrangements in the Weyl arrangements of type $D_{n}$ which admit rank 3 restrictions which fail to be free, cf. [AMR18, Rem. 3.6]. In particular, accuracy is not hereditary in general. See also Example 5.7 for such an instance in a graphic arrangement.

(iv) Also accuracy is not preserved under taking localizations, see Examples 5.7 and 5.15.

(v) A product of arrangements is accurate if and only if each factor is accurate, cf. [OT92, Prop. 2.14, Prop. 4.28].

Our methods actually apply in a slightly more general setting. Instead of considering only MAT-free arrangements, i.e. arrangements build up from the empty arrangement using Theorem 2.10, we can instead start with a suitable free arrangement (which need not be empty) and add hyperplanes successively by means of Theorem 2.10 (Theorem 3.11).

Utilizing this fact together with a recent observation by Abe and Terao from [AT19], and a special case of a result by the same authors from [AT16] gives our fourth main theorem, demonstrating that yet another prominent class of arrangements is accurate, namely the so called ideal-Shi arrangements $\mathrm{Shi}_{\mathcal{I}}^{k}$ and the extended Catalan arrangements $\mathrm{Cat}^{k}$ (Definition 4.6). For details, see Section 4.2.

Theorem 1.8. Extended Shi arrangements $\mathrm{Shi}^{k}$, ideal-Shi arrangements $\mathrm{Shi}_{\mathcal{I}}^{k}$ and extended Catalan arrangements $\mathrm{Cat}^{k}$ are accurate. 
In Section 5.1, we study arrangements that satisfy a slightly weaker property, so called almost accurate arrangements, see Definition 5.1, where compared to accurate arrangements, we simply disregard the ordering of the exponents involved. This property in turn is easily seen to be implied by many well established concepts of freeness such as supersolvability or divisional freeness, for instance.

However, in contrast it turns out that in general, accuracy is not implied by supersolvability (so neither by inductive freeness nor by divisional freeness), see Example 5.4.

On the other hand there are accurate arrangements which are not divisionally free, see Example 5.5. So in particular, accuracy is not implied by divisional freeness in general. Consequently, the recent result by Cuntz, Röhrle and Schauenburg [CRS19], asserting that all ideal arrangements are divisionally free does not imply Theorem 1.3.

In Section 5.2, we consider free graphic arrangements under the aspect of accuracy. In Example 5.7, we present a non-accurate free graphic arrangement which admits an extension by one vertex which gives an accurate graphic arrangement. This example also shows that accuracy is neither preserved under localizations nor under restrictions.

In view of the original theme of Orlik, Solomon and Terao for Coxeter arrangements (Theorem 1.6), we consider complex reflection arrangements and their restrictions in our final Section 5.3, and determine all accurate members among them (see Theorems 5.8 and 5.12). Along the way we show that accuracy from our motivating case of Coxeter arrangements (Theorem 1.6) descends to restrictions of the latter (Corollary 5.14).

For general information about arrangements, Weyl groups and root systems, we refer the reader to [Bou68] and [OT92].

\section{PReliminaries}

2.1. Hyperplane arrangements. Let $\mathscr{A}=(\mathscr{A}, V)$ be a hyperplane arrangement in $V \cong \mathbb{K}^{\ell}$ where $\mathbb{K}=\mathbb{R}$ or $\mathbb{K}=\mathbb{C}$. If we want to emphasize the dimension $\ell$ of the ambient vector space we say that $\mathscr{A}$ is an $\ell$-arrangement. The intersection lattice of $\mathscr{A}$ is $L(\mathscr{A})=$ $\left\{\cap_{H \in \mathscr{B}} H \mid \mathscr{B} \subseteq \mathscr{A}\right\}$.

Associated with $X \in L(\mathscr{A})$ we have two canonical arrangements, the localization $\mathscr{A}_{X}$ of $\mathscr{A}$ at $X$, given by

$$
\mathscr{A}_{X}:=\{H \in \mathscr{A} \mid H \supseteq X\},
$$


and the restriction $\mathscr{A}^{X}$ of $\mathscr{A}$ to $X$, defined by

$$
\mathscr{A}^{X}:=\left\{H \cap X \mid H \in \mathscr{A} \backslash \mathscr{A}_{X}\right\} \text {. }
$$

The rank of $X \in L(\mathscr{A})$ is defined as $\operatorname{rk}(X):=\ell-\operatorname{dim}(X)$ and the rank of $\mathscr{A}$ as $\operatorname{rk}(\mathscr{A})=\operatorname{rk}\left(\cap_{H \in \mathscr{A}} H\right)$.

Let $S=S\left(V^{*}\right)$ be the symmetric algebra of the dual space $V^{*}$. We fix a basis $x_{1}, \ldots, x_{\ell}$ for $V^{*}$ and identify $S$ with the polynomial ring $\mathbb{K}\left[x_{1}, \ldots, x_{\ell}\right]$. The algebra $S$ is equipped with the grading by polynomial degree: $S=\bigoplus_{p \in \mathbb{Z}} S_{p}$, where $S_{p}$ is the $\mathbb{K}$-space of homogeneous polynomials of degree $p$ (along with 0 ), where $S_{p}=\{0\}$ for $p<0$.

2.2. Free arrangements. A $\mathbb{K}$-linear map $\theta: S \rightarrow S$ which satisfies $\theta(f g)=\theta(f) g+f \theta(g)$ is called a $\mathbb{K}$-derivation. Let $\operatorname{Der}(S)$ be the $S$-module of $\mathbb{K}$-derivations of $S$. It is a free $S$-module with basis $\partial / \partial x_{1}, \ldots, \partial / \partial x_{\ell}$. We say that $\theta \in \operatorname{Der}(S)$ is homogeneous of degree $p$ provided $\theta=\sum_{i=1}^{\ell} f_{i} \partial / \partial x_{i}$ with $f_{i} \in S_{p}$ for each $1 \leq i \leq \ell$. In this case we write $\operatorname{deg} \theta=p$. We obtain a $\mathbb{Z}$-grading $\operatorname{Der}(S)=\bigoplus_{p \in \mathbb{Z}} \operatorname{Der}(S)_{p}$ of the $S$-module $\operatorname{Der}(S)$.

Definition 2.1. For $H \in \mathscr{A}$ we fix $\alpha_{H} \in V^{*}$ with $H=\operatorname{ker}\left(\alpha_{H}\right)$. The module of $\mathscr{A}$-derivations is defined by

$$
D(\mathscr{A}):=\left\{\theta \in \operatorname{Der}(S) \mid \theta\left(\alpha_{H}\right) \in \alpha_{H} S \text { for all } H \in \mathscr{A}\right\} .
$$

In particular, if $\mathscr{B} \subseteq \mathscr{A}$, then $D(\mathscr{A}) \subseteq D(\mathscr{B})$.

We say that $\mathscr{A}$ is free if the module of $\mathscr{A}$-derivations is a free $S$ module.

Let $X \in L(\mathscr{A})$ be of $\operatorname{rank} q$ and let $P_{X}:=\left\langle\alpha_{H} \mid H \in \mathscr{A}_{X}\right\rangle_{S}$ be the prime ideal generated by the defining linear forms of the localization $\mathscr{A}_{X}$. After a possible base change we may assume that $X=\operatorname{ker}\left(x_{\ell-q+1}\right) \cap \ldots \cap \operatorname{ker}\left(x_{\ell}\right)$. Hence $P_{X}=\left\langle x_{\ell-q+1}, \ldots, x_{\ell}\right\rangle_{S}$ and then $S^{X}:=S / P_{X} \cong \mathbb{K}\left[x_{1}, \ldots, x_{\ell-q}\right]$. The module $D\left(\mathscr{A}^{X}\right)$ is naturally an $S^{X}$-submodule of $\operatorname{Der}\left(S^{X}\right)$. For $\theta \in D(\mathscr{A})$ we have $\theta\left(P_{X}\right) \subseteq P_{X}$ and hence we get a restriction map

$$
D(\mathscr{A}) \rightarrow D\left(\mathscr{A}^{X}\right), \theta \mapsto \theta^{X} \quad \text { by } \quad \theta^{X}\left(f+P_{X}\right)=\theta(f)+P_{X} .
$$

The derivation $\theta^{X} \in D\left(\mathscr{A}^{X}\right)$ is the restriction of $\theta$ to $X$, cf. [OT92, $\S 4.3]$. Moreover, if $\theta$ is homogeneous of degree $\operatorname{deg} \theta=d$ and $\theta^{X} \neq 0$ then $\operatorname{deg}\left(\theta^{X}\right)=d$.

If $\mathscr{A}$ is a free arrangement we may choose a homogeneous basis $\theta_{1}, \ldots, \theta_{\ell}$ of $D(\mathscr{A})$. Then the degrees of the $\theta_{i}$ are called the exponents of $\mathscr{A}$. They are uniquely determined by $\mathscr{A}$, [OT92, Def. 4.25]. In that case we write

$$
\exp (\mathscr{A}):=\left(\operatorname{deg} \theta_{1}, \ldots, \operatorname{deg} \theta_{\ell}\right)
$$


for the exponents of $\mathscr{A}$.

Note that the empty arrangement $\varnothing_{\ell}$ in $V$ is free with $D\left(\varnothing_{\ell}\right)=$ $\operatorname{Der}(S)$ so that $\exp \left(\varnothing_{\ell}\right)=(0, \ldots, 0) \in \mathbb{Z}^{\ell}$.

If $\theta_{1}, \ldots, \theta_{\ell} \in \operatorname{Der}(S)$ then

$$
M\left(\theta_{1}, \ldots, \theta_{\ell}\right):=\left(\theta_{j}\left(x_{i}\right)\right)_{1 \leq i, j \leq \ell}
$$

denotes the coefficient matrix of the $\theta_{i}$, i.e. the matrix of coefficients with respect to the standard basis $\partial / \partial x_{1}, \ldots, \partial / \partial x_{\ell}$ of $\operatorname{Der}(S)$.

Let $\left\{\alpha_{H} \mid H \in \mathscr{A}\right\}$ be defining linear forms for the hyperplanes in $\mathscr{A}$. Then

$$
Q(\mathscr{A})=\prod_{H \in \mathscr{A}} \alpha_{H} \in S
$$

is the defining polynomial of $\mathscr{A}$. It is uniquely determined by $\mathscr{A}$ up to a non-zero constant factor.

Next we recall Saito's criterion, cf. [OT92, Thm. 4.19].

Theorem 2.2. For $\theta_{1}, \ldots, \theta_{\ell} \in D(\mathscr{A})$, the following are equivalent:

(1) $\operatorname{det}\left(M\left(\theta_{1}, \ldots, \theta_{\ell}\right)\right) \in \mathbb{K}^{\times} Q(\mathscr{A})$,

(2) $\theta_{1}, \ldots, \theta_{\ell}$ is a basis of $D(\mathscr{A})$.

The corollary to the following result from $[\mathrm{OT} 92, \S 4]$ provides a convenient degree criterion to determine when a derivation of a deletion $\mathscr{A} \backslash\{H\}$ of $\mathscr{A}$ does belong to the smaller $S$-module $D(\mathscr{A})$.

Proposition 2.3 ([OT92, Prop. 4.41]). Let $H=\operatorname{ker}(\alpha) \in \mathscr{A}, \mathscr{A}^{\prime}=$ $\mathscr{A} \backslash\{H\}$ and $\mathscr{A}^{\prime \prime}=\mathscr{A}^{H}$. Then there is a homogeneous polynomial $b$ in $S$ of degree $\left|\mathscr{A}^{\prime}\right|-\left|\mathscr{A}^{\prime \prime}\right|$ such that

$$
\theta(\alpha) \in \alpha S+b S
$$

for all $\theta \in D\left(\mathscr{A}^{\prime}\right)$.

Corollary 2.4. With the notation as in Proposition 2.3, if $\theta \in D\left(\mathscr{A}^{\prime}\right)$ with $\operatorname{deg}(\theta)<\operatorname{deg}(b)=\left|\mathscr{A}^{\prime}\right|-\left|\mathscr{A}^{\prime \prime}\right|$ then $\theta \in D(\mathscr{A})$.

We require a construction from $[\mathrm{OT} 92, \S 4]$ that allows us to extend $S$-independent members from $D(\mathscr{A})$ to a basis of $D(\mathscr{A})$ in case $\mathscr{A}$ is free. Here and later on we write $\left(e_{1}, \ldots, e_{\ell}\right) \leq$ for $\left(e_{1} \leq e_{2} \leq \ldots \leq e_{\ell}\right)$.

Proposition 2.5 ([OT92, Thm. 4.42]). Let $\mathscr{A}$ be a free arrangement with $\exp (\mathscr{A})=\left(e_{1}, \ldots, e_{\ell}\right)_{\leq}$, and let $\theta_{1}, \ldots, \theta_{k} \in D(\mathscr{A})$ be homogeneous elements such that

(1) $\operatorname{deg}\left(\theta_{i}\right)=e_{i}$

(2) $\theta_{i} \notin S \theta_{1}+\ldots+S \theta_{i-1}$.

Then $\theta_{1}, \ldots, \theta_{k}$ may be extended to a basis of $D(\mathscr{A})$. 
We finally recall the restriction part of Terao's seminal AdditionDeletion Theorem [Ter80a].

Proposition 2.6 ([OT92, Cor. 4.47]). Let $H \in \mathscr{A}$ and $\mathscr{A}^{\prime}=\mathscr{A} \backslash\{H\}$ such that both $\mathscr{A}$ and $\mathscr{A}^{\prime}$ are free. Then $\mathscr{A}^{H}$ is free and we have

$$
\begin{aligned}
\exp (\mathscr{A}) & =\left(e_{1}, \ldots, e_{\ell-1}, e_{\ell}\right), \\
\exp \left(\mathscr{A}^{\prime}\right) & =\left(e_{1}, \ldots, e_{\ell-1}, e_{\ell}-1\right), \\
\exp \left(\mathscr{A}^{H}\right) & =\left(e_{1}, \ldots, e_{\ell-1}\right) .
\end{aligned}
$$

2.3. Topological incarnation of the module of $\mathscr{A}$-derivations. The complement of $\mathscr{A}=(\mathscr{A}, V)$ is denoted by

$$
M(\mathscr{A}):=V \backslash \bigcup_{H \in \mathscr{A}} H .
$$

For $z \in V$ let $T_{V, z}$ be the tangent space of $V$ at $z$; it is a $\mathbb{K}$-vector space with basis $\partial / \partial x_{1}, \ldots, \partial / \partial x_{\ell}$. The $\mathbb{K}$-linear evaluation map

$$
\rho_{z}: D(\mathscr{A}) \rightarrow T_{V, z}
$$

is defined as follows, cf. [OT92, §5.1]. For $\theta=\sum_{i=1}^{\ell} f_{i} \partial / \partial x_{i} \in D(\mathscr{A})$, let

$$
\rho_{z}(\theta):=\sum_{i=1}^{\ell} f_{i}(z) \partial / \partial x_{i} .
$$

Proposition 2.7 ([OT92, Prop. 5.17]). For $z \in M\left(\mathscr{A}^{X}\right)$, we have $\rho_{z}(D(\mathscr{A}))=T_{X, z}$ which is isomorphic to $X$ as a $\mathbb{K}$-vector space.

Corollary 2.8. If $\mathscr{A}$ is free, $\theta_{1}, \ldots, \theta_{\ell}$ is a basis of $D(\mathscr{A})$, and $z \in$ $M(\mathscr{A})$, then

$$
\rho_{z}(D(\mathscr{A}))=T_{V, z}=\left\langle\rho_{z}\left(\theta_{1}\right), \ldots, \rho_{z}\left(\theta_{\ell}\right)\right\rangle_{\mathbb{K}}
$$

i.e. $\rho_{z}\left(\theta_{1}\right), \ldots, \rho_{z}\left(\theta_{\ell}\right)$ is a $\mathbb{K}$-vector space basis of $T_{V, z}$.

Proof. Note that for $\theta=\sum_{i=1}^{\ell} f_{i} \theta_{i}$ we have $\rho_{z}(\theta)=\sum_{i=1}^{\ell} f_{i}(z) \rho_{z}\left(\theta_{i}\right)$. By Proposition 2.7, the $\mathbb{K}$-linear map $\rho_{z}$ is onto $T_{V, z}$ and since $\theta_{1}, \ldots, \theta_{\ell}$ is a basis of $D(\mathscr{A})$, for every $v \in T_{V, z}$ there are $f_{1}, \ldots, f_{\ell} \in S$ such that $v=\rho_{z}\left(\sum_{i=1}^{\ell} f_{i} \theta_{i}\right)=\sum_{i=1}^{\ell} f_{i}(z) \rho_{z}\left(\theta_{i}\right)$. Consequently, $\rho_{z}\left(\theta_{1}\right), \ldots, \rho_{z}\left(\theta_{\ell}\right)$ is a basis of the vector space $T_{V, z}$.

The next fact provides a central part in the proof of Theorem 1.2.

Proposition 2.9. Let $\mathscr{B} \subseteq \mathscr{A}$ be free arrangements. Assume that $\theta_{1}, \ldots, \theta_{\ell}$ is a basis of $D(\mathscr{B})$ such that $\theta_{i} \in D(\mathscr{A})$ for $1 \leq i \leq \ell-q$. Suppose $X \in L(\mathscr{A})$ such that $\mathscr{A}_{X} \cap \mathscr{B}=\varnothing$. Then $\operatorname{rk}(X) \leq q$. 
Proof. Since $\mathscr{A}_{X} \cap \mathscr{B}=\varnothing$, we see that $M\left(\mathscr{A}^{X}\right) \cap M(\mathscr{B}) \neq \varnothing$. Let $z \in$ $M\left(\mathscr{A}^{X}\right) \cap M(\mathscr{B})$. Then thanks to Proposition 2.7, we have $\rho_{z}(D(\mathscr{A}))=$ $T_{X, z}$. But also the tangent vectors $\rho_{z}\left(\theta_{1}\right), \ldots, \rho_{z}\left(\theta_{\ell}\right)$ form a vector space basis of $T_{V, z}$, by Corollary 2.8. By our assumption the $\theta_{i}$ belong to $D(\mathscr{A})$ for $1 \leq i \leq \ell-q$. So $\rho_{z}\left(\theta_{1}\right), \ldots, \rho_{z}\left(\theta_{\ell-q}\right) \in T_{X, z}$ and they are linearly independent. Hence $\operatorname{dim}(X)=\operatorname{dim}\left(T_{X, z}\right) \geq \ell-q$, i.e., $\operatorname{rk}(X) \leq q$.

2.4. MAT-free arrangements. We begin by recalling the core result from $\left[\mathrm{ABC}^{+} 16\right]$, the so-called Multiple Addition Theorem (MAT).

Theorem $2.10\left(\left[\mathrm{ABC}^{+} 16\right.\right.$, Thm. 3.1]). Let $\mathscr{A}^{\prime}=\left(\mathscr{A}^{\prime}, V\right)$ be a free arrangement with $\exp \left(\mathscr{A}^{\prime}\right)=\left(e_{1}, \ldots, e_{\ell}\right) \leq$ and let $1 \leq p \leq \ell$ be the multiplicity of the highest exponent, i.e.

$$
e_{\ell-p}<e_{\ell-p+1}=\cdots=e_{\ell}=: e .
$$

Let $H_{1}, \ldots, H_{q}$ be hyperplanes in $V$ with $H_{i} \notin \mathscr{A}^{\prime}$ for $i=1, \ldots, q$. Define

$$
\mathscr{A}_{j}^{\prime \prime}:=\left(\mathscr{A}^{\prime} \cup\left\{H_{j}\right\}\right)^{H_{j}}=\left\{H \cap H_{j} \mid H \in \mathscr{A}^{\prime}\right\}, \quad \text { for } j=1, \ldots, q .
$$

Assume that the following conditions are satisfied:

(1) $X:=H_{1} \cap \cdots \cap H_{q}$ is q-codimensional.

(2) $X \nsubseteq \bigcup_{H \in \mathscr{A}^{\prime}} H$.

(3) $\left|\mathscr{A}^{\prime}\right|-\left|\mathscr{A}_{j}^{\prime \prime}\right|=$ e for $1 \leq j \leq q$.

Then $q \leq p$ and $\mathscr{A}:=\mathscr{A}^{\prime} \cup\left\{H_{1}, \ldots, H_{q}\right\}$ is free with

$$
\exp (\mathscr{A})=\left(e_{1}, \ldots, e_{\ell-q}, e+1, \ldots, e+1\right)_{\leq} .
$$

We often consider the addition of several hyperplanes using Theorem 2.10. This motivates the next terminology.

Definition 2.11. Let $\mathscr{A}^{\prime}$ and $\left\{H_{1}, \ldots, H_{q}\right\}$ be as in Theorem 2.10 such that conditions (1)-(3) are satisfied. Then the addition of $\left\{H_{1}, \ldots, H_{q}\right\}$ to $\mathscr{A}^{\prime}$ resulting in $\mathscr{A}=\mathscr{A}^{\prime} \cup\left\{H_{1}, \ldots, H_{q}\right\}$ is called an MAT-step.

The following lemma about a certain basis of the derivation module across an MAT-step follows directly from the last lines of the proof of Theorem 2.10 (cf. $\left[\mathrm{ABC}^{+} 16\right.$, Thm. 3.1]).

Lemma 2.12. Let $\mathscr{A}^{\prime}$ be free with $\exp \left(\mathscr{A}^{\prime}\right)=\left(e_{1}, \ldots, e_{\ell-p}, e, \ldots, e\right)_{\leq}$ and let $\mathscr{A}=\mathscr{A}^{\prime} \dot{\cup}\left\{H_{1}=\operatorname{ker}\left(\alpha_{1}\right), \ldots, H_{p}=\operatorname{ker}\left(\alpha_{p}\right)\right\}$ be an MAT-step.

Then there is a basis $\theta_{1}, \ldots, \theta_{\ell-p}, \eta_{1}, \ldots, \eta_{p}$ of $D\left(\mathscr{A}^{\prime}\right)$ with $\operatorname{deg}\left(\theta_{i}\right)=$ $e_{i}$ for $1 \leq i \leq \ell-p$ and $\operatorname{deg}\left(\eta_{j}\right)=e$ for $1 \leq j \leq p$, such that for any subset $N \subseteq\{1, \ldots, p\}$ and its complement $\bar{N}=\{1, \ldots, p\} \backslash N$, the derivations

$$
\theta_{1}, \ldots, \theta_{\ell-p},\left\{\alpha_{j} \eta_{j}\right\}_{j \in \bar{N}},\left\{\eta_{i}\right\}_{i \in N}
$$


form a basis of $D\left(\mathscr{A}^{\prime} \cup\left\{H_{j} \mid j \in \bar{N}\right\}\right)$. In particular, $\mathscr{A}^{\prime} \cup\left\{H_{j} \mid j \in \bar{N}\right\}$ is free.

As a consequence of Lemma 2.12 and a successive application of Proposition 2.6 we obtain the following.

Corollary 2.13. Let $\mathscr{A}^{\prime}$ be free, $\mathscr{A}=\mathscr{A}^{\prime} \dot{\cup}\left\{H_{1}, \ldots, H_{p}\right\}$ an MAT-step and $\mathscr{C} \subseteq\left\{H_{1}, \ldots, H_{p}\right\}$. Suppose that $\exp (\mathscr{A})=\left(e_{1}, \ldots, e_{\ell}\right)_{\leq}$and let $X:=\cap_{H \in \mathscr{C}} H$. Then $\mathscr{A}^{X}$ is free with $\exp \left(\mathscr{A}^{X}\right)=\left(e_{1}, \ldots, e_{\ell-|\mathscr{C}|}\right)_{\leq}$.

An iterative application of Theorem 2.10 motivates the following natural concept.

Definition 2.14 ([CM20, Def. 3.2, Lem. 3.8]). An arrangement $\mathscr{A}$ is called MAT-free if there exists an ordered partition

$$
\pi=\left(\pi_{1}|\cdots| \pi_{n}\right)
$$

of $\mathscr{A}$ such that the following hold. Set $\mathscr{A}_{0}:=\varnothing_{\ell}$ and

$$
\mathscr{A}_{k}:=\bigcup_{i=1}^{k} \pi_{i} \quad \text { for } 1 \leq k \leq n .
$$

Then for every $0 \leq k \leq n-1$ suppose that

(1) $\operatorname{rk}\left(\pi_{k+1}\right)=\left|\pi_{k+1}\right|$,

(2) $\cap_{H \in \pi_{k+1}} H \nsubseteq \bigcup_{H^{\prime} \in \mathscr{A}_{k}} H^{\prime}$,

(3) $\left|\mathscr{A}_{k}\right|-\left|\left(\mathscr{A}_{k} \cup\{H\}\right)^{H}\right|=k$ for each $H \in \pi_{k+1}$,

i.e. $\mathscr{A}_{k+1}=\mathscr{A}_{k} \cup \pi_{k+1}$ is an MAT-step.

An ordered partition $\pi$ with these properties is called an MATpartition for $\mathscr{A}$.

Note that in [CM20] MAT-freeness was defined differently. However, for our purpose its characterization in [CM20, Lem. 3.8] is sufficient. Hence we take the latter here for our definition.

Remark 2.15. Suppose that $\mathscr{A}$ is MAT-free with MAT-partition $\pi=$ $\left(\pi_{1}|\cdots| \pi_{n}\right)$. Then we have:

(a) for each $1 \leq k \leq n, \mathscr{A}_{k}$ is MAT-free with MAT-partition $\left(\pi_{1}|\cdots| \pi_{k}\right)$,

(b) $\mathscr{A}$ is free and the exponents $\exp (\mathscr{A})=\left(e_{1}, \ldots, e_{\ell}\right)_{\leq}$of $\mathscr{A}$ are given

by the block sizes of the dual partition of $\pi$ :

$$
e_{i}:=\left|\left\{k|| \pi_{k} \mid \geq \ell-i+1\right\}\right|,
$$

(c) $\left|\pi_{1}\right|>\left|\pi_{2}\right| \geq \cdots \geq\left|\pi_{n}\right|$.

Proof. Statement (a) is clear by Definition 2.14. Statements (b) and

(c) follow readily from Theorem 2.10 and a simple induction. 


\section{Proof of Theorem 1.2}

We begin with a closer investigation of MAT-free arrangements. The idea of our proof is to control a basis of the module of $\mathscr{A}$-derivations through MAT-steps and to apply Proposition 2.9.

This in turn leads to the identification of a restricted subarrangement with a restriction of the whole arrangement. Since the former is free with the right exponents, by Corollary 2.13, the freeness of the restriction of the entire arrangement follows.

3.1. Basis derivations across MAT-steps. First, we recall a technical result which also provided a key step in the proof of Theorem 2.10 (cf. $\left[\mathrm{ABC}^{+} 16\right.$, p. 1343]).

Lemma 3.1. Let $\mathscr{A}^{\prime}$ be free with $\exp \left(\mathscr{A}^{\prime}\right)=\left(e_{1}, \ldots, e_{\ell-p}, e, \ldots, e\right)_{<}$, $e_{\ell-p}<e$, and $\mathscr{A}=\mathscr{A}^{\prime} \cup\left\{H_{1}=\operatorname{ker}\left(\alpha_{1}\right), \ldots, H_{r}=\operatorname{ker}\left(\alpha_{r}\right)\right\}$ be an $M A \bar{T}-$ step. Let $\theta_{1}, \ldots, \theta_{\ell-p}, \varphi_{1}, \ldots, \varphi_{p}$ be a homogeneous basis of $D\left(\mathscr{A}^{\prime}\right)$ with $\operatorname{deg}\left(\theta_{i}\right)=e_{i}$ for $1 \leq i \leq \ell-p$ and $\operatorname{deg}\left(\varphi_{i}\right)=e$ for $1 \leq i \leq p$.

Then there are a matrix $C=\left(c_{i j}\right) \in \mathbb{K}^{p \times r}$ of rank $r$ and homogeneous polynomials $b_{j}$ of degree $e$, for $1 \leq j \leq r$, such that

$$
\varphi_{i}\left(\alpha_{j}\right) \equiv c_{i j} b_{j} \quad \bmod \alpha_{j} S \quad \text { for } 1 \leq i \leq p, 1 \leq j \leq r .
$$

With this lemma we are able to maintain a certain part of a given basis of the derivation modules while performing MAT-steps.

Lemma 3.2. Suppose that $\mathscr{A}^{\prime}$ is free with $\exp \left(\mathscr{A}^{\prime}\right)=\left(e_{1}, \ldots, e_{\ell-p}\right.$, $e, \ldots, e)_{\leq}, e_{\ell-p}<e$, and that $\mathscr{A}=\mathscr{A}^{\prime} \dot{\cup}\left\{H_{1}, \ldots, H_{r}\right\}$ is an MATstep. Let $\theta_{1}, \ldots, \theta_{\ell-p}, \varphi_{1}, \ldots, \varphi_{p}$ be a homogeneous basis of $D\left(\mathscr{A}^{\prime}\right)$ with $\operatorname{deg}\left(\theta_{i}\right)=e_{i}$ for $1 \leq i \leq \ell-p$ and $\operatorname{deg}\left(\varphi_{i}\right)=e$ for $1 \leq i \leq p$.

Then for each $r \leq q \leq p$ there are a subset $N \subseteq\{1, \ldots, p\}$ with $|N|=q$ and $\psi_{j} \in D(\mathscr{A})$, for $j \in \bar{N}=\{1, \ldots, p\} \backslash N$, such that

$$
\theta_{1}, \ldots, \theta_{\ell-p},\left\{\psi_{j}\right\}_{j \in \bar{N}},\left\{\varphi_{i}\right\}_{i \in N}
$$

form a basis of $D\left(\mathscr{A}^{\prime}\right)$.

Proof. By Lemma 3.1 there are a matrix $C=\left(c_{i j}\right) \in \mathbb{K}^{p \times r}$ of rank $r$ and homogeneous polynomials $b_{j}$ of degree $e$, for $1 \leq j \leq q$, such that

$$
\varphi_{i}\left(\alpha_{j}\right) \equiv c_{i j} b_{j} \quad \bmod \alpha_{j} S \quad \text { for } 1 \leq i \leq p, 1 \leq j \leq r .
$$

So for $r \leq q \leq p$ there is a subset $N=\left\{i_{1}, \ldots, i_{q}\right\} \subseteq\{1, \ldots, p\}$ such that the $q$ rows $\left(c_{i 1}, \ldots, c_{i r}\right)$, for $i \in N$ of the matrix $C$ contain a basis of the whole row space of $C$. Now, by elementary row operations there is a regular matrix $T \in \mathrm{GL}_{p}(\mathbb{K})$ such that the transformation of the 
basis given by $T$ fixes the derivations $\varphi_{i}$ for $i \in N$ :

$$
\begin{aligned}
& \left(\varphi_{1}, \ldots, \varphi_{p}\right) \cdot T \\
= & \left(\psi_{1}, \ldots, \psi_{i_{1}-1}, \varphi_{i_{1}}, \psi_{i_{1}+1}, \ldots, \psi_{i_{q}-1}, \varphi_{i_{q}}, \psi_{i_{q}+1}, \ldots, \psi_{p}\right),
\end{aligned}
$$

and

$$
\psi_{i}\left(\alpha_{j}\right) \equiv 0 \quad \bmod \alpha_{j} S \quad \text { for all } i \in \bar{N}, 1 \leq j \leq r,
$$

i.e. $\psi_{i} \in D(\mathscr{A})$ for all $i \in \bar{N}$. We further have

$$
\begin{aligned}
& \operatorname{det}\left(M\left(\theta_{1}, \ldots, \theta_{\ell-p},\left\{\psi_{j}\right\}_{j \in \bar{N}},\left\{\varphi_{i}\right\}_{i \in N}\right)\right) \\
= & \operatorname{det}(T) \operatorname{det}\left(M\left(\theta_{1}, \ldots, \theta_{\ell-p}, \varphi_{1}, \ldots, \varphi_{p}\right)\right) .
\end{aligned}
$$

Since $\theta_{1}, \ldots, \theta_{\ell-p}, \varphi_{1}, \ldots, \varphi_{p}$ form a basis of $D\left(\mathscr{A}^{\prime}\right)$ and $\operatorname{det}(T) \in \mathbb{K}^{\times}$, by Theorem $2.2, \theta_{1}, \ldots, \theta_{\ell-p},\left\{\psi_{j}\right\}_{j \in \bar{N}},\left\{\varphi_{i}\right\}_{i \in N}$ is again a basis of $D\left(\mathscr{A}^{\prime}\right)$, as claimed.

The following technical proposition provides a crucial step in the proof of Theorem 1.2.

Proposition 3.3. Let $\mathscr{B}^{\prime}$ be free with $\exp \left(\mathscr{B}^{\prime}\right)=\left(e_{1}, \ldots, e_{\ell-p}, e, \ldots, e\right)_{\leq}$. Suppose that

(a) $\mathscr{A}^{\prime}=\mathscr{B}^{\prime} \cup\left\{H_{1}, \ldots, H_{p}\right\}$ is an MAT-step such that

$$
\exp \left(\mathscr{A}^{\prime}\right)=\left(e_{1}, \ldots, e_{\ell-p}, e+1, \ldots, e+1\right)_{\leq},
$$

(b) $\mathscr{A}=\mathscr{A}^{\prime} \dot{\cup}\left\{K_{1}, \ldots, K_{r}\right\}$ is an MAT-step such that

$$
\exp (\mathscr{A})=\left(e_{1}, \ldots, e_{\ell-p}, e+1, \ldots, e+1, e+2, \ldots, e+2\right)_{\leq} .
$$

Then for each $r \leq q \leq p$ there are $a \mathscr{C} \subseteq\left\{H_{1}, \ldots, H_{p}\right\}$ with $|\mathscr{C}|=q$ and a basis $\theta_{1}, \ldots, \theta_{\ell}$ of $D(\mathscr{B})$, where $\mathscr{B}=\mathscr{A}^{\prime} \backslash \mathscr{C}$, such that $\theta_{i} \in D(\mathscr{A})$ for $1 \leq i \leq \ell-q$, $\operatorname{deg}\left(\theta_{i}\right)=e_{i}$ for $1 \leq i \leq \ell-p$, and $\operatorname{deg}\left(\theta_{j}\right)=e+1$ for $\ell-p+1 \leq j \leq \ell-q$.

Proof. Let $H_{1}=\operatorname{ker}\left(\alpha_{1}\right), \ldots, H_{p}=\operatorname{ker}\left(\alpha_{p}\right)$. By Lemma 2.12 applied to the first MAT-step $\mathscr{A}^{\prime}=\mathscr{B}^{\prime} \cup\left\{H_{1}, \ldots, H_{p}\right\}$, there exists a homogeneous basis $\theta_{1}, \ldots, \theta_{\ell-p}, \eta_{1}, \ldots, \eta_{p}$ of $D\left(\mathscr{B}^{\prime}\right)$ with $\operatorname{deg}\left(\theta_{i}\right)=e_{i}, 1 \leq i \leq \ell-p$ and $\operatorname{deg}\left(\eta_{j}\right)=e, 1 \leq j \leq p$ such that $\theta_{1}, \ldots, \theta_{\ell-p}, \alpha_{1} \eta_{1}, \ldots, \alpha_{p} \eta_{p}$ form a homogeneous basis of $D\left(\mathscr{A}^{\prime}\right)$.

Note that, since $\operatorname{deg} \theta_{i}<e+1=\left|\mathscr{A}^{\prime}\right|-\left|\left(\mathscr{A}^{\prime} \cup\left\{K_{j}\right\}\right)^{K_{j}}\right|$ (by condition (3) of Theorem 2.10 within the second MAT-step), we have $\theta_{i} \in D(\mathscr{A})$ for $1 \leq i \leq \ell-p$, by Corollary 2.4 .

Thanks to Lemma 3.2, applied to the second MAT-step, for each $r \leq q \leq p$, there are a subset $N \subseteq\{1, \ldots, p\}$ with $|N|=q$ and derivations $\psi_{j} \in D(\mathscr{A})$, for $j \in \bar{N}=\{1, \ldots, p\} \backslash N$ such that $\theta_{1}, \ldots, \theta_{\ell-p}$, $\left\{\psi_{j}\right\}_{j \in \bar{N}},\left\{\alpha_{i} \eta_{i}\right\}_{i \in N}$ form a basis of $D\left(\mathscr{A}^{\prime}\right)$. 
Next we define

$$
\mathscr{C}:=\left\{H_{i} \mid i \in N\right\} \quad \text { and } \quad \mathscr{B}:=\mathscr{A}^{\prime} \backslash \mathscr{C},
$$

and we set

$$
\begin{aligned}
\left\{\theta_{\ell-p+1}, \ldots, \theta_{\ell-q}\right\} & :=\left\{\psi_{j} \mid j \in \bar{N}\right\}, \text { and } \\
\left\{\theta_{\ell-q+1}, \ldots, \theta_{\ell}\right\} & :=\left\{\eta_{i} \mid i \in N\right\} .
\end{aligned}
$$

According to Lemma 2.12 applied to the first MAT-step, we observe that $\theta_{1}, \ldots, \theta_{\ell-p},\left\{\alpha_{j} \eta_{j}\right\}_{j \in \bar{N}},\left\{\eta_{i}\right\}_{i \in N}$ form a basis of $D(\mathscr{B})$. In particular, we have $\eta_{i} \in D(\mathscr{B})$ for $i \in N$.

Then, since $Q(\mathscr{C})=\prod_{i \in N} \alpha_{i}$, and by the multilinearity of the determinant and Theorem 2.2, we conclude

$$
\begin{aligned}
& \operatorname{det}\left(M\left(\theta_{1}, \ldots, \theta_{\ell}\right)\right)= \frac{\operatorname{det}\left(M\left(\theta_{1}, \ldots, \theta_{\ell-p},\left\{\psi_{j}\right\}_{j \in \bar{N}},\left\{\alpha_{i} \eta_{i}\right\}_{i \in N}\right)\right)}{Q(\mathscr{C})} \\
& \in \mathbb{K}^{\times} \frac{Q\left(\mathscr{A}^{\prime}\right)}{Q(\mathscr{C})}=\mathbb{K}^{\times} Q(\mathscr{B}) .
\end{aligned}
$$

Consequently, since $\psi_{j} \in D(\mathscr{A}) \subseteq D(\mathscr{B})$, for $j \in \bar{N}$, all derivations $\theta_{1}, \ldots, \theta_{\ell}$ do belong to $D(\mathscr{B})$ and thus they provide the desired basis of $D(\mathscr{B})$, again by Theorem 2.2.

From the proof of Proposition 3.3 we record the following:

Corollary 3.4. Let $\mathscr{A}^{\prime}, \mathscr{A}, \mathscr{B}$, and $q$ be as in Proposition 3.3. Let $\mathscr{C}=$ $\left\{\operatorname{ker}\left(\beta_{1}\right), \ldots, \operatorname{ker}\left(\beta_{q}\right)\right\}$. Then there is a basis $\theta_{1}, \ldots, \theta_{\ell-q}, \eta_{1}, \ldots, \eta_{q}$ of $D(\mathscr{B})$ such that $\theta_{1}, \ldots, \theta_{\ell-q}, \beta_{1} \eta_{1}, \ldots, \beta_{q} \eta_{q}$ is a basis of $D\left(\mathscr{A}^{\prime}\right), \operatorname{deg} \theta_{i}=$ $e_{i}$, and $\theta_{i} \in D(\mathscr{A})$ for $1 \leq i \leq \ell-q$.

3.2. Restrictions of MAT-free arrangements. For this subsection, let $\mathscr{A}$ be an MAT-free arrangement with MAT-partition $\pi=$ $\left(\pi_{1}|\cdots| \pi_{n}\right)$. Recall from Definition 2.14 that for $k \in\{1, \ldots, n\}$ we set $\mathscr{A}_{k}:=\bigcup_{i=1}^{k} \pi_{i}$ and $\pi_{j}=\emptyset$ for $j>n$.

Our next result is used to maintain a certain part of a basis of the module of derivations of a subarrangement and also later to construct a particular basis of $D(\mathscr{A})$.

Lemma 3.5. Fix $k \in\{1, \ldots, n\}$. If $\theta \in D\left(\mathscr{A}_{k}\right)$ is homogeneous with $\operatorname{deg}(\theta)<k$, then $\theta \in D(\mathscr{A})$.

Proof. For each of the MAT-steps $\mathscr{A}_{s+1}=\mathscr{A}_{s} \cup \pi_{s+1}, k \leq s \leq n-1$, we have $\operatorname{deg}(\theta)<k \leq s=\left|\mathscr{A}_{s}\right|-\left|\left(\mathscr{A}_{s} \cup\{H\}\right)^{H}\right|$ for each $H \in \pi_{s+1}$ (by condition (3) of Theorem 2.10). Hence $\theta \in D\left(\mathscr{A}_{s+1}\right)$, by Corollary 2.4, and ultimately we get $\theta \in D(\mathscr{A})$.

Our next result provides the right setting to apply Proposition 2.9. 
Proposition 3.6. Fix $k \in\{1, \ldots, n\}$. For each $\left|\pi_{k+1}\right| \leq q \leq\left|\pi_{k}\right|$ there are a $\mathscr{C} \subseteq \pi_{k}$ with $|\mathscr{C}|=q$ and a basis $\theta_{1}, \ldots, \theta_{\ell}$ of $D\left(\mathscr{A}_{k} \backslash \mathscr{C}\right)$ such that $\theta_{1}, \ldots, \theta_{\ell-q} \in D(\mathscr{A})$.

Proof. For this proof, set $p_{j}:=\left|\pi_{j}\right|$ for all $j \geq 0$.

First let $k=n$. Then by Lemma 2.12 applied to the MAT-step $\mathscr{A}=\mathscr{A}_{n-1} \cup \pi_{n}$, there is a basis $\theta_{1}, \ldots, \theta_{\ell-p_{n}}, \eta_{1}, \ldots, \eta_{p_{n}}$ of $D\left(\mathscr{A}_{n-1}\right)$ such that the derivations $\theta_{1}, \ldots, \theta_{\ell-p_{n}}, \alpha_{1} \eta_{1}, \ldots, \alpha_{p_{n}} \eta_{p_{n}}$ form a basis of $D(\mathscr{A})$. Further, for each $p_{n+1}=0 \leq q \leq p_{n}$, set $\mathscr{C}:=$ $\left\{\operatorname{ker}\left(\alpha_{p_{n}-q+1}\right), \ldots, \operatorname{ker}\left(\alpha_{p_{n}}\right)\right\} \subseteq \pi_{n}$, and define

$$
\theta_{\ell-p_{n}+1}:=\alpha_{1} \eta_{1}, \ldots, \theta_{\ell-q}:=\alpha_{p_{n}-q} \eta_{p_{n}-q} \in D(\mathscr{A}),
$$

and $\theta_{\ell-q+1}:=\eta_{p_{n}-q+1}, \ldots, \theta_{\ell}:=\eta_{p_{n}}$. Then by yet another application of Lemma 2.12, we get that $\theta_{1}, \ldots, \theta_{\ell}$ form a basis of $D\left(\mathscr{A}_{n} \backslash \mathscr{C}\right)$ such that $\theta_{1}, \ldots, \theta_{\ell-q} \in D(\mathscr{A})$.

Now suppose $k<n$. Then for the two MAT-steps $\mathscr{A}_{k}=\mathscr{A}_{k-1} \cup \pi_{k}$ and $\mathscr{A}_{k+1}=\mathscr{A}_{k} \cup \pi_{k+1}$ we are precisely in the situation of Proposition 3.3. Suppose that $\exp \left(\mathscr{A}_{k}\right)=\left(e_{1}, \ldots, e_{\ell-p_{k}}, k, \ldots, k\right)_{<}$. So by the statement of the lemma, for each $p_{k+1} \leq q \leq p_{k}$ there exists a $\mathscr{C} \subseteq \pi_{k}$ with $|\mathscr{C}|=q$ and a basis $\theta_{1}, \ldots, \theta_{\ell}$ of $D\left(\mathscr{A}_{k} \backslash \mathscr{C}\right)$ such that $\theta_{i} \in D\left(\mathscr{A}_{k+1}\right)$ for $1 \leq i \leq \ell-q, \operatorname{deg}\left(\theta_{i}\right)=e_{i} \leq k$ for $1 \leq i \leq \ell-p_{k}$, and $\operatorname{deg}\left(\theta_{j}\right)=k$ for $\ell-p_{k}+1 \leq j \leq \ell-q$.

Finally, since $\operatorname{deg}\left(\theta_{i}\right)<k+1$ for $1 \leq i \leq \ell-q$, we also have $\theta_{i} \in D(\mathscr{A})$ for $1 \leq i \leq \ell-q$, by Lemma 3.5.

The following corollary to the preceding proposition is the key to the proof of Theorem 1.2.

Corollary 3.7. Let $k, q$ and $\mathscr{C}$ be as in Proposition 3.6. Let $X \in L(\mathscr{A})$ with $\mathscr{A}_{X} \cap\left(\mathscr{A}_{k} \backslash \mathscr{C}\right)=\varnothing$. Then $\operatorname{rk}(X) \leq q$.

Proof. Owing to Proposition 3.6, there is a basis $\theta_{1}, \ldots, \theta_{\ell}$ of $D\left(\mathscr{A}_{k} \backslash \mathscr{C}\right)$ such that $\theta_{1}, \ldots, \theta_{\ell-q} \in D(\mathscr{A})$. Since $X \in L(\mathscr{A})$ with $\mathscr{A}_{X} \cap\left(\mathscr{A}_{k} \backslash \mathscr{C}\right)=$ $\varnothing$, we readily obtain $\operatorname{rk}(X) \leq q$, according to Proposition 2.9.

Next we define a surjective map from a subarrangement to the restriction of the ambient arrangement.

Lemma 3.8. Let $k, q$ and $\mathscr{C}$ be as in Proposition 3.6. Set $X:=$ $\cap_{H \in \mathscr{C}} H$. Then the map

$$
\begin{aligned}
\mathscr{A}_{k} \backslash \mathscr{C} & \rightarrow \mathscr{A}^{X} \\
H^{\prime} & \mapsto H^{\prime} \cap X
\end{aligned}
$$

is surjective. In particular, $\left(\mathscr{A}_{k}\right)^{X}=\mathscr{A}^{X}$. 
Proof. Set $\mathscr{B}:=\mathscr{A}_{k} \backslash \mathscr{C}$. Let $Y=\tilde{H} \cap X \in \mathscr{A}^{X}$ for some $\tilde{H} \in \mathscr{A}$. In particular, $\operatorname{rk}(Y)=\operatorname{rk}(X)+1=q+1>q$. Thus we have $\mathscr{A}_{Y} \cap \mathscr{B} \neq \varnothing$, by Corollary 3.7. Further, by the conditions (1) and (2) of Theorem 2.10, we have $\mathscr{B}_{X}=\varnothing$. So there is an $H^{\prime} \in\left(\mathscr{A}_{Y} \backslash \mathscr{A}_{X}\right) \cap \mathscr{B}$ such that $Y=H^{\prime} \cap X$.

Armed with the various results about MAT-steps from above, we are finally in a position to attack Theorem 1.2.

Theorem 3.9. Let $\mathscr{A}$ be an MAT-free arrangement with MAT-partition $\pi=\left(\pi_{1}|\cdots| \pi_{n}\right)$ and exponents $\exp (\mathscr{A})=\left(e_{1}, \ldots, e_{\ell}\right)_{\leq}$. Then for each $1 \leq k \leq n$ and each $\left|\pi_{k+1}\right| \leq q \leq\left|\pi_{k}\right|$ there is a $\mathscr{C} \subseteq \pi_{k}$ with $|\mathscr{C}|=q$ such that for $X:=\cap_{H \in \mathscr{C}} H$ the restriction $\mathscr{A}^{X}$ is free with exponents

$$
\exp \left(\mathscr{A}^{X}\right)=\left(e_{1}, \ldots, e_{\ell-q}\right)_{\leq}
$$

Furthermore, there is a basis $\theta_{1}, \ldots, \theta_{\ell}$ of $D(\mathscr{A})$ such that $\theta_{1}^{X}, \ldots, \theta_{\ell-q}^{X}$ is a basis of $D\left(\mathscr{A}^{X}\right)$.

Proof. First note that $\mathscr{A}_{k}=\mathscr{A}_{k-1} \cup \pi_{k}$ is an MAT-step. Thanks to Corollary 2.13 the restriction $\left(\mathscr{A}_{k}\right)^{X}$ is free with $\exp \left(\left(\mathscr{A}_{k}\right)^{X}\right)=$ $\left(e_{1}, \ldots, e_{\ell-q}\right)_{\leq}$. Now we also have $\mathscr{A}^{X}=\left(\mathscr{A}_{k}\right)^{X}$, by Lemma 3.8, and so the first statement follows.

Now suppose $\mathscr{C}=\left\{\operatorname{ker}\left(\beta_{1}\right), \ldots, \operatorname{ker}\left(\beta_{q}\right)\right\}$. To derive the existence of the particular basis compatible with restriction, observe that there is a basis $\theta_{1}, \ldots, \theta_{\ell-q}, \eta_{1}, \ldots, \eta_{q}$ of $D\left(\mathscr{A}_{k} \backslash \mathscr{C}\right)$ with $\operatorname{deg}\left(\theta_{i}\right)=e_{i}, \theta_{i} \in$ $D\left(\mathscr{A}_{k+1}\right)$ for $1 \leq i \leq \ell-q$ so that $\theta_{1}, \ldots, \theta_{\ell-q}, \beta_{1} \eta_{1}, \ldots, \beta_{q} \eta_{q}$ form a basis of $D\left(\mathscr{A}_{k}\right)$, owing to Corollary 3.4. Thanks to condition (1) in Theorem 2.10 the linear forms $\beta_{1}, \ldots, \beta_{q}$ are linearly independent. Using the same arguments as the ones in the proof of [OT92, Thm. 4.46] successively along with a simple induction on $q$, the restrictions $\theta_{i}^{X}$ of the $\theta_{i}$ to $X$ for $1 \leq i \leq \ell-q$ yield a basis of $D\left(\mathscr{A}_{k}^{X}\right)=D\left(\mathscr{A}^{X}\right)$.

Considering the degrees of the $\theta_{i}$, we have $\theta_{1}, \ldots, \theta_{\ell-q} \in D(\mathscr{A})$, by Lemma 3.5. Now thanks to Proposition 2.5, we may extend $\theta_{1}, \ldots, \theta_{\ell-q}$ to a complete basis $\theta_{1}, \ldots, \theta_{\ell-q}, \theta_{\ell-q+1}, \ldots, \theta_{\ell}$ of $D(\mathscr{A})$. This finishes the proof.

Corollary 3.10 (Theorem 1.2). MAT-free arrangements are accurate.

3.3. A generalization of Theorem 1.2. In this section, we record an extension of Theorem 3.9. It can be applied to obtain the accuracy of not necessarily MAT-free arrangements (which we do in Section 4.2). Instead of considering MAT-free arrangements which are build up from the empty arrangement using MAT-steps, we can more generally study arrangements which are build up from some suitable free arrangement 
(which need not be empty) via MAT-steps. We formalize this in the following manner.

Let $\mathscr{A}^{\prime}=\left(\mathscr{A}^{\prime}, V\right)$ be a free arrangement with exponents $\exp \left(\mathscr{A}^{\prime}\right)=$ $\left(e_{1}, \ldots, e_{\ell-p}, e, \ldots, e\right)_{\leq} \in \mathbb{Z}^{\ell}$. Let $\mathscr{B}=(\mathscr{B}, V)$ be an arrangement in $V$ disjoint from $\mathscr{A}$ and partitioned by $\pi=\left(\pi_{1}|\cdots| \pi_{n}\right)$. Set $\mathscr{A}_{0}:=\mathscr{A}^{\prime}$,

$$
\mathscr{A}_{k}:=\mathscr{A}^{\prime} \dot{\cup}\left(\cup_{i=1}^{k} \pi_{i}\right) \text {, }
$$

and $\pi_{j}=\emptyset$ for $j>n$. Suppose that $\mathscr{A}_{k} \cup \pi_{k+1}$ is an MAT-step for $0 \leq k \leq n-1$. Then $\mathscr{A}=\mathscr{A}^{\prime} \cup \mathscr{B}$ is free by a successive application of Theorem 2.10. In this setting, all arguments from Sections 3.1 and 3.2 apply and Theorem 3.9 directly generalizes in the following fashion.

Theorem 3.11. Let $\mathscr{A}=\mathscr{A}^{\prime} \dot{\cup} \mathscr{B}$ be a free arrangement obtained from the free arrangement $\mathscr{A}^{\prime}$ through $M A T$-steps with exponents $\exp (\mathscr{A})=$ $\left(e_{1}, \ldots, e_{\ell}\right)_{\leq}$. Suppose that $\pi=\left(\pi_{1}|\cdots| \pi_{n}\right)$ is the corresponding ordered partition of $\mathscr{B}$. Then for each $1 \leq k \leq n$ and each $\left|\pi_{k+1}\right| \leq q \leq$ $\left|\pi_{k}\right|$ there is a $\mathscr{C} \subseteq \pi_{k}$ with $|\mathscr{C}|=q$ such that for $X:=\cap_{H \in \mathscr{C}} H$ the restriction $\mathscr{A}^{X}$ is free with exponents

$$
\exp \left(\mathscr{A}^{X}\right)=\left(e_{1}, \ldots, e_{\ell-q}\right)_{\leq}
$$

Furthermore, there is a basis $\theta_{1}, \ldots, \theta_{\ell}$ of $D(\mathscr{A})$ such that $\theta_{1}^{X}, \ldots, \theta_{\ell-q}^{X}$ is a basis of $D\left(\mathscr{A}^{X}\right)$.

As a corollary to Theorem 3.11 we get the following sufficient condition for accuracy.

Corollary 3.12. Let $\mathscr{A}, \mathscr{B}$ and $\pi$ be as in Theorem 3.11. If $\left|\pi_{1}\right| \geq \ell-2$ then $\mathscr{A}$ is accurate.

We conclude this section with an example which shows that the ordered partition $\pi=\left(\pi_{1}|\cdots| \pi_{n}\right)$ of the subarrangement $\mathscr{B} \subseteq \mathscr{A}$ in Theorem 3.11 is itself not an MAT-partiton for $\mathscr{B}$ and $\mathscr{B}$ does not even need to be free, in general.

Example 3.13. Let $\mathscr{A}$ be the 3-arrangement with defining polynomial

$$
Q(\mathscr{A})=x_{1} x_{2} x_{3}\left(x_{1}-x_{2}\right)\left(x_{1}-x_{3}\right)\left(x_{2}-x_{3}\right)\left(x_{2}+x_{3}\right) .
$$

In particular, $\mathscr{A}$ is an ideal subarrangement of the Weyl arrangement of type $B_{3}$, see Section 4.1. Then $\mathscr{A}^{\prime}=\left\{\operatorname{ker}\left(x_{1}-x_{2}\right), \operatorname{ker}\left(x_{2}-\right.\right.$ $\left.\left.x_{3}\right), \operatorname{ker}\left(x_{3}\right)\right\}, \mathscr{B}=\mathscr{A} \backslash \mathscr{A}^{\prime}$ and $\pi=\left(\pi_{1}, \pi_{2}\right)$ with

$$
\begin{aligned}
& \pi_{1}=\left\{\operatorname{ker}\left(x_{1}-x_{3}\right), \operatorname{ker}\left(x_{2}\right)\right\}, \\
& \pi_{2}=\left\{\operatorname{ker}\left(x_{2}+x_{3}\right), \operatorname{ker}\left(x_{1}\right)\right\},
\end{aligned}
$$

apparently satisfy all the assumptions of Theorem 3.11. But here, $\mathscr{B}$ is a generic 3-arrangement with four hyperplanes and as such, it is 
not even free, e.g. by Terao's Factorization Theorem [Ter81], as its characteristic polynomial has non-integral roots.

\section{Applications}

In this section we apply our main Theorem 3.9 and its generalization, Theorem 3.11 to Weyl arrangements, their ideal subarrangements, extended Catalan arrangements and ideal-Shi arrangements.

4.1. Weyl and Ideal arrangements. For general information about Weyl groups and their root systems, see [Bou68].

Let $W$ be a Weyl group acting as a reflection group on the real vector space $V=\mathbb{R}^{\ell}$. Let $\Phi:=\Phi(W) \subseteq V^{*}$ be a (reduced) root system for $W$ and $\Phi^{+} \subseteq \Phi$ a positive system with simple roots $\Delta \subseteq \Phi^{+}$. The $\operatorname{rank}$ of $W$ respectively $\Phi$ is $\operatorname{rk}(W):=\operatorname{rk}(\Phi):=\operatorname{dim} \mathbb{R} \Phi$. We have $\Phi^{+}=\left(\sum_{\alpha \in \Delta} \mathbb{Z}_{\geq 0} \alpha\right) \cap \Phi$, i.e. if $\beta \in \Phi^{+}$then there are integers $n_{\alpha} \in \mathbb{Z}_{\geq 0}$ such that $\beta=\sum_{\alpha \in \Delta} n_{\alpha} \alpha$. Then the height of $\beta$ is defined by

$$
\operatorname{ht}(\beta):=\sum_{\alpha \in \Delta} n_{\alpha}
$$

The partial order $\leq$ on $\Phi^{+}$is defined by

$$
\beta \leq \gamma: \Longleftrightarrow \gamma-\beta \in \sum_{\alpha \in \Delta} \mathbb{Z}_{\geq 0} \alpha
$$

A subset $\mathcal{I} \subseteq \Phi^{+}$is an ideal if it is a (lower) order ideal in the poset $\left(\Phi^{+}, \leq\right)$, i.e. for $\alpha \in \mathcal{I}$ and $\beta \in \Phi^{+}$with $\beta \leq \alpha$, we have $\beta \in \mathcal{I}$.

The Weyl arrangement $\mathscr{A}(W)$ is the hyperplane arrangement in $V$ defined by

$$
\mathscr{A}(W):=\left\{\operatorname{ker}(\beta) \mid \beta \in \Phi^{+}\right\} .
$$

Definition 4.1 ([ABC $\left.\left.{ }^{+} 16\right]\right)$. If $\mathcal{I} \subseteq \Phi^{+}$is an order ideal then

$$
\mathscr{A}_{\mathcal{I}}:=\{\operatorname{ker}(\beta) \mid \beta \in \mathcal{I}\} \subseteq \mathscr{A}(W)
$$

is called an ideal (sub)arrangement.

We denote by $m_{\mathcal{I}}$ the maximal height of a root in $\mathcal{I}$. For $1 \leq k \leq m_{\mathcal{I}}$, let

$$
\pi_{k, \mathcal{I}}:=\{\operatorname{ker}(\alpha) \mid \alpha \in \mathcal{I}, \operatorname{ht}(\alpha)=k\}
$$

and let

$$
\pi_{\mathcal{I}}:=\left(\pi_{1, \mathcal{I}}|\cdots| \pi_{m_{\mathcal{I}}, \mathcal{I}}\right)
$$

be the root-height partition of $\mathscr{A}_{\mathcal{I}}$. Set $\pi_{j, \mathcal{I}}=\emptyset$ for $j>m_{\mathcal{I}}$.

Next we recall the principal result from $\left[\mathrm{ABC}^{+} 16\right.$, Thm. 1.1] (Idealfree Theorem) in our terminology, using the notation above. 


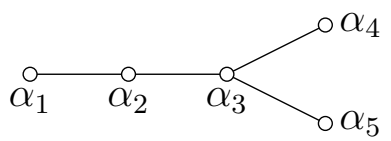

Figure 1. The Dynkin diagram of $D_{5}$ with the corresponding numbering of the simple roots.

Theorem 4.2. Let $\mathscr{A}_{\mathcal{I}} \subseteq \mathscr{A}(W)$ be an ideal subarrangement of the Weyl arrangement $\mathscr{A}(W)$ for the order ideal $\mathcal{I} \subseteq \Phi^{+}$. Then $\mathscr{A}_{\mathcal{I}}$ is MAT-free with MAT-partition $\pi_{\mathcal{I}}=\left(\pi_{1, \mathcal{I}}|\cdots| \pi_{m_{\mathcal{I}}, \mathcal{I}}\right)$ and exponents

$$
\exp \left(\mathscr{A}_{\mathcal{I}}\right)=\left(e_{1}^{\mathcal{I}}, \ldots, e_{\ell}^{\mathcal{I}}\right)
$$

where

$$
e_{r}^{\mathcal{I}}=\left|\left\{j|| \pi_{j, \mathcal{I}} \mid \geq \ell-r+1\right\}\right| .
$$

Combining Theorems 3.9 and 4.2 and using the notation from above, we immediately obtain the following.

Theorem 4.3. Let $\mathscr{A}=\mathscr{A}_{\mathcal{I}} \subseteq \mathscr{A}(W)$ be an ideal subarrangement of the Weyl arrangement $\mathscr{A}(W)$ for the order ideal $\mathcal{I} \subseteq \Phi^{+}$.

Then for each $1 \leq k \leq m_{\mathcal{I}}$ and $\left|\pi_{k+1, \mathcal{I}}\right| \leq q \leq\left|\pi_{k, \mathcal{I}}\right|$ there is a $\mathscr{C} \subseteq \pi_{k, \mathcal{I}}$ with $|\mathscr{C}|=q$ such that for $X:=\cap_{H \in \mathscr{C}} H$ the restriction $\mathscr{A}^{X}$ is free with exponents

$$
\exp \left(\mathscr{A}^{X}\right)=\left(e_{1}^{\mathcal{I}}, \ldots, e_{\ell-q}^{\mathcal{I}}\right),
$$

where

$$
e_{r}^{\mathcal{I}}=\left|\left\{j|| \pi_{j, \mathcal{I}} \mid \geq \ell-r+1\right\}\right| .
$$

Corollary 4.4 (Theorem 1.3). Ideal arrangements are accurate.

Example 4.5. Let $\Phi$ be the root system of type $D_{5}$ and write $c_{1} c_{2} c_{3} c_{c_{5}}$ for $\beta=\sum_{i} c_{i} \alpha_{i} \in \Phi^{+}$with the numbering of the simple roots according to the Dynkin diagram displayed in Figure 1. Let $\mathcal{I}:=\left\{\beta \in \Phi^{+} \mid \beta \leq\right.$ $012_{1}^{1}$, or $\beta \leq 111_{0}^{1}$, or $\left.\beta \leq 111_{1}^{0}\right\}$ be the ideal as shown in Figure 2 .

Then $m_{\mathcal{I}}=0+1+2+1+1=5$ and $\mathscr{A}_{\mathcal{I}}$ is MAT-free with MATpartition $\left(\pi_{1, \mathcal{I}}|\cdots| \pi_{5, \mathcal{I}}\right)$ and exponents $\exp \left(\mathscr{A}_{\mathcal{I}}\right)=(1,3,4,4,5)$.

Now, for $Y:=\operatorname{ker}\left(010_{0}^{0}\right) \cap \operatorname{ker}\left(001_{0}^{0}\right)$ the characteristic polynomial of the restriction $\mathscr{A}_{\mathcal{I}}^{Y}$ does not factor over $\mathbb{Z}$ and consequently, the restriction $\mathscr{A}_{\mathcal{I}}^{Y}$ is not free by Terao's Factorization Theorem [Ter81].

But by Theorem 4.3 for e.g. $\mathscr{C}=\left\{\operatorname{ker}\left(111_{1}^{0}\right), \operatorname{ker}\left(011_{1}^{1}\right)\right\} \subseteq \pi_{4, \mathcal{I}}$ and $X=\cap_{H \in \mathscr{C}} H=\operatorname{ker}\left(111_{1}^{0}\right) \cap \operatorname{ker}\left(011_{1}^{1}\right)$, the restriction $\mathscr{A}_{\mathcal{I}}^{X}$ is free with exponents $\exp \left(\mathscr{A}_{\mathcal{I}}^{X}\right)=(1,3,4)$. Note that the exponents can be easily 

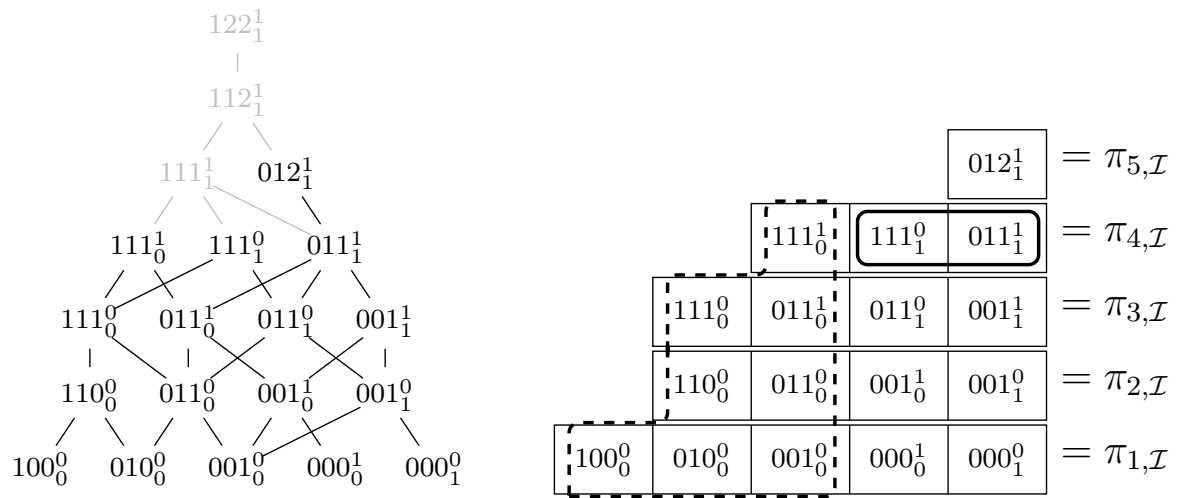

Figure 2. The root-poset of type $D_{5}$, the height partition of the ideal $\mathcal{I}$, and a restriction of the ideal subarrangement $\mathscr{A}_{\mathcal{I}} \subseteq \mathscr{A}\left(D_{5}\right)$.

read off the enclosed part (dashed line) of the partition diagram as illustrated in Figure 2.

The preceding example shows that Theorem 4.3 is probably the strongest general statement one can give concerning the freeness of restrictions of ideal arrangements and their exponents.

4.2. Ideal-Shi and extended Catalan arrangements. Let $W, V$ and $\Delta \subseteq \Phi^{+} \subseteq \Phi \subseteq V^{*}$ be as in Section 4.1. Let

$$
h:=m_{\Phi^{+}}+1
$$

be the Coxeter number of $W$. Embed $V \subseteq V^{\prime}=\mathbb{R}^{\ell+1}$ and let $z \in$ $\left(V^{\prime}\right)^{*} \backslash\{0\}$ such that $V=\operatorname{ker}(z)=: H_{z}$, i.e. $z$ corresponds to the $(\ell+1)$ st coordinate. For $j \in \mathbb{Z}$ define

$$
H_{\alpha}^{j}:=\operatorname{ker}(\alpha-j z) \text {. }
$$

The combinatorics of deformations of Weyl arrangements was first studied by Athanasiadis in [Ath96], among them the so called extended Shi arrangements. We are interested in the following generalization, investigated by Abe and Terao in [AT16], the so called ideal-Shi arrangements.

Definition 4.6. For $k \in \mathbb{Z}_{>0}$ the extended Shi arrangements are defined as

$$
\mathrm{Shi}^{k}:=\left\{H_{\alpha}^{j} \mid-k+1 \leq j \leq k\right\} \cup\left\{H_{z}\right\} .
$$

For $\mathcal{I} \subseteq \Phi^{+}$an ideal, the ideal-Shi arrangements are defined as

$$
\operatorname{Shi}_{\mathcal{I}}^{k}:=\operatorname{Shi}^{k} \cup\left\{H_{\beta}^{-k} \mid \beta \in \mathcal{I}\right\} .
$$


In the special case when $\mathcal{I}=\Phi^{+}$we obtain the extended Catalan arrangements

$$
\mathrm{Cat}^{k}:=\mathrm{Shi}_{\Phi^{+}}^{k} \cdot
$$

In [Yos04], Yoshinaga proved the following remarkable theorem, confirming a conjecture by Edelman and Reiner [ER96, Conj. 3.3].

Theorem 4.7 ([Yos04, Thm. 1.2]). Let $k \in \mathbb{Z}_{>0}$. Then the extended Shi arrangement $\mathrm{Shi}^{k}$ is free with exponents

$$
\exp \left(\mathrm{Shi}^{k}\right)=(1, h k, \ldots, h k) \in \mathbb{Z}^{\ell+1},
$$

and the extended Catalan arrangement $\mathrm{Cat}^{k}$ is free with exponents

$$
\exp \left(\mathrm{Cat}^{k}\right)=\left(1, h k+e_{1}, \ldots, h k+e_{\ell}\right),
$$

where $\left(e_{1}, \ldots, e_{\ell}\right)=\exp (\mathscr{A}(W))$.

We need the following special case of a result by Abe and Terao (cf. [AT16, Thm. 1.6]).

Proposition 4.8. Let $\Sigma \subseteq \Delta$ be a subset of the simple roots of $\Phi^{+}$. Then the arrangement

$$
\mathrm{Shi}_{-\Sigma}^{k}:=\mathrm{Shi}^{k} \backslash\left\{H_{\alpha}^{k} \mid \alpha \in \Sigma\right\}
$$

is free with

$$
\exp \left(\mathrm{Shi}_{-\Sigma}^{k}\right)=(1, h k-1, \ldots, h k-1, h k, \ldots, h k),
$$

where the multiplicity of the exponent $h k-1$ is $|\Sigma|$.

Next we observe that in the case $\Sigma=\Delta$, rejoining the hyperplanes $H_{\alpha}^{k}$ for $\alpha \in \Delta$ to $\mathrm{Shi}_{-\Delta}^{k}$ is an MAT-step.

Lemma 4.9. The addition $\mathrm{Shi}^{k}=\mathrm{Shi}_{-\Delta}^{k} \cup\left\{H_{\alpha}^{k} \mid \alpha \in \Delta\right\}$ is an MATstep.

Proof. We have to verify conditions (1)-(3) from Theorem 2.10. Condition (1) is clear, since the simple roots $\Delta$ are linearly independent and so are the linear forms $\alpha-k z$ for $\alpha \in \Delta$. Condition (3) follows from Propositions 2.6 and 4.8 and the fact that the sum of the exponents of a free arrangement coincides with its cardinality, cf. [OT92, Thm. 4.23].

Finally, we need to show condition (2), i.e. that $X=\cap_{\alpha \in \Delta} H_{\alpha}^{k} \nsubseteq H$ for any $H=H_{\beta}^{j} \in \mathrm{Shi}_{-\Delta}^{k}$. For a contradiction, we assume the contrary. That is, there are an index $-k+1 \leq j \leq k$ and a positive $\operatorname{root} \beta \in \Phi^{+}$ such that

$$
\beta-j z=\sum_{\alpha \in \Delta} n_{\alpha}(\alpha-k z)
$$


for suitable $n_{\alpha}$. But then $\beta=\sum_{\alpha \in \Delta} n_{\alpha} \alpha$, so $n_{\alpha} \in \mathbb{Z}_{\geq 0}$ for all $\alpha \in \Delta$ and $n_{\alpha} \geq 1$ for some $\alpha \in \Delta$ since $\beta$ is a positive root. Therefore,

$$
j=\sum_{\alpha \in \Delta} n_{\alpha} k \geq k
$$

Hence we must have $j=k$ and thus $\beta$ belongs to $\Phi^{+} \backslash \Delta$. Consequently, in the expression $\beta=\sum_{\alpha \in \Delta} n_{\alpha} \alpha$ at least two coefficients must be positive. But then $k=j \geq 2 k$, which is absurd, as $k>0$ in Definition 4.6 .

In [AT19, Sec. 5], Abe and Terao showed that an ideal-Shi arrangement and in particular an extended Catalan arrangement can be constructed via MAT-steps starting from the corresponding extended Shi arrangement. Hence we are exactly in the setting of Theorem 3.11 with $\mathscr{A}^{\prime}=\mathrm{Shi}_{-\Delta}^{k}, \mathscr{B}=\left\{H_{\alpha}^{k} \mid \alpha \in \Delta\right\} \cup\left\{H_{\beta}^{-k} \mid \beta \in \mathcal{I}\right\}$ and

$$
\pi=\pi_{\mathcal{I}}^{k}:=\left(\pi_{1, \mathcal{I}}^{k}|\cdots| \pi_{m_{\mathcal{I}}+1, \mathcal{I}}^{k}\right)
$$

where

$$
\begin{aligned}
\pi_{1, \mathcal{I}}^{k} & =\left\{H_{\alpha}^{k} \mid \alpha \in \Delta\right\}, \text { and } \\
\pi_{t+1, \mathcal{I}}^{k} & :=\left\{H_{\beta}^{-k} \mid \beta \in \mathcal{I}, \operatorname{ht}(\beta)=t\right\},
\end{aligned}
$$

for $1 \leq t \leq m_{\mathcal{I}}$. Set $\pi_{j, \mathcal{I}}^{k}=\emptyset$ for $j>m_{\mathcal{I}}+1$. Then the arguments from the proofs in [AT19, Sec. 5], together with Lemma 4.9 and Theorem 3.11 readily imply the following.

Theorem 4.10. Let $\mathrm{Shi}_{\mathcal{I}}^{k}$ be an ideal-Shi arrangement for the order ideal $\mathcal{I} \subseteq \Phi^{+}$. Then for each $1 \leq t \leq m_{\mathcal{I}}+1$ and each $\left|\pi_{t+1, \mathcal{I}}^{k}\right| \leq q \leq$ $\left|\pi_{t, \mathcal{I}}^{k}\right|$ there is a $\mathscr{C} \subseteq \pi_{t, \mathcal{I}}^{k}$ with $|\mathscr{C}|=q$ such that for $X:=\cap_{H \in \mathscr{C}} H$ the restriction $\left(\mathrm{Shi}_{\mathcal{I}}^{k}\right)^{X}$ is free with exponents

$$
\exp \left(\left(\operatorname{Shi}_{\mathcal{I}}^{k}\right)^{X}\right)=\left(1, h k+e_{1}^{\mathcal{I}}, \ldots, h k+e_{\ell-q}^{\mathcal{I}}\right)_{\leq},
$$

where

$$
e_{r}^{\mathcal{I}}=\left|\left\{j|| \pi_{j+1, \mathcal{I}}^{k} \mid \geq \ell-r+1\right\}\right| .
$$

We close this section with the following special case of Theorem 4.10.

Corollary 4.11 (Theorem 1.8). Extended Shi arrangements $\mathrm{Shi}^{k}$ and ideal-Shi arrangements $\mathrm{Shi}_{\mathcal{I}}^{k}$ are accurate. In particular, extended Catalan arrangements $\mathrm{Cat}^{k}$ are accurate. 


\section{Complements And Examples}

In our final section we discuss other notions of freeness in relation to accuracy.

Further we also study accurate graphic arrangements. Here we show that accuracy is neither preserved under taking localizations nor under restrictions.

Finally, in view of Theorem 1.6, we consider accuracy among complex reflection arrangements and their restrictions.

5.1. Almost accurate arrangements. We begin by weakening the property from Definition 1.1 by dropping the condition that the exponents of the free restriction $\mathscr{A}^{X}$ match those of $\mathscr{A}$ ordered by size.

Definition 5.1. An arrangement $\mathscr{A}$ is said to be almost accurate if $\mathscr{A}$ is free with exponents $\exp (\mathscr{A})=\left(e_{1}, e_{2}, \ldots, e_{\ell}\right)$ and for every $1 \leq d \leq \ell$ there exists an intersection $X$ of hyperplanes from $\mathscr{A}$ of dimension $d$ such that $\mathscr{A}^{X}$ is free with $\exp \left(\mathscr{A}^{X}\right) \subseteq \exp (\mathscr{A})$.

Clearly, if $\mathscr{A}$ is accurate then it is almost accurate. In particular, by Theorem 1.2, MAT-free arrangements are almost accurate. However, the converse is false, see Example 5.4.

Note that a product of arrangements is almost accurate if and only if each factor is almost accurate, by [OT92, Prop. 2.14, Prop. 4.28].

Next we indicate that there are several natural classes of free arrangements that are almost accurate.

For an arrangement $\mathscr{A}$ we denote its characteristic polynomial by $\chi(\mathscr{A}, t)$, see [OT92, Sec. 2.3]. Next we recall the key result from [Abe16].

Theorem 5.2 ([Abe16, Thm. 1.1]). Suppose there is a hyperplane $H$ in $\mathscr{A}$ such that the restriction $\mathscr{A}^{H}$ is free and that $\chi\left(\mathscr{A}^{H}, t\right)$ divides $\chi(\mathscr{A}, t)$. Then $\mathscr{A}$ is free.

Theorem 5.2 can be viewed as a strengthening of the addition part of Terao's Theorem [Ter80a] ([OT92, Thm. 4.51]). An iterative application leads to the class of divisionally free arrangements:

Definition 5.3 ([Abe16, Def. 1.5]). An arrangement $\mathscr{A}$ is called divisionally free if either $\ell \leq 2, \mathscr{A}=\varnothing_{\ell}$, or else there is a flag of subspaces $X_{i}$ of rank $i$ in $L(\mathscr{A})$

$$
X_{0}=V \supset X_{1} \supset X_{2} \supset \cdots \supset X_{\ell-2}
$$

so that $\chi\left(\mathscr{A}^{X_{i}}, t\right)$ divides $\chi\left(\mathscr{A}^{X_{i-1}}, t\right)$, for $i=1, \ldots, \ell-2$. 
It is clear from Theorem 5.2, Definition 5.3, and the fact that rank 2 arrangements are free, that divisionally free arrangements are almost accurate. However, the converse is false, see Example 5.5. Nevertheless, since supersolvable arrangements are inductively free ([OT92, Thm. 4.58]) and the latter are divisionally free ([Abe16, Thm. 1.6]), there is an abundance of arrangements that are almost accurate.

It was already observed in [CM20] that MAT-freeness and divisional freeness are closely related. Thus, in view of Theorem 1.2 , it is natural to investigate the relation between divisional freeness and accuracy. However, Examples 5.4 and 5.5 below do show that there are supersolvable (whence divisionally free) arrangements that are not accurate and vice versa.

Example 5.4. Let $\mathscr{A}=\mathscr{A}(14,1)$ be the supersolvable simplicial arrangement of rank 3 with 14 hyperplanes, cf. [Grü09], [CM19]. We have $\mathscr{A}=\left\{H_{1}, \ldots, H_{7}, K_{1}, \ldots, K_{7}\right\}, X=\cap_{i=1}^{7} H_{i}$ is modular of rank 2 , and $X \nsubseteq K_{j}$ for all $1 \leq j \leq 7$. Consider $\mathscr{B}:=\mathscr{A} \backslash\left\{K_{1}, K_{2}\right\}$. Since $\mathscr{B}$ is obtained from $\mathscr{A}$ by removing hyperplanes away from the modular element $X, \mathscr{B}$ is still supersolvable and, thanks to [OT92, Thm. 4.58], $\mathscr{B}$ is also free with exponents $\exp (\mathscr{B})=(1,5,6)$. Using the explicit description of $\mathscr{A}$ from [CM19], one easily checks that $\exp \left(\mathscr{B}^{H}\right)$ belongs to $\{(1,3),(1,4),(1,6)\}$ for $H \in \mathscr{B}$. Consequently, $\mathscr{B}$ is not accurate.

Example 5.5. In [HR19] a free arrangement $\mathscr{D}$ is constructed within a rank 5 restriction of the Weyl arrangement of type $E_{7}$ which is not divisionally free with exponents $(1,5,5,5,5)$ and defining polynomial

$$
\begin{aligned}
Q(\mathscr{D})= & x_{2}\left(x_{1}+x_{3}-x_{5}\right)\left(2 x_{1}+x_{2}+x_{3}\right)\left(2 x_{1}+x_{2}+2 x_{3}+x_{4}-x_{5}\right) \\
& x_{5}\left(x_{1}+x_{3}\right)\left(x_{2}+x_{5}\right)\left(2 x_{1}+x_{2}+2 x_{3}+x_{4}\right)\left(2 x_{1}+x_{3}-x_{5}\right) \\
& \left(2 x_{1}+2 x_{2}+2 x_{3}+x_{4}\right)\left(x_{2}+x_{3}+x_{4}\right)\left(x_{1}+x_{2}+x_{3}+x_{4}\right) \\
& \left(x_{3}+x_{4}\right)\left(x_{1}+x_{2}+x_{3}\right) x_{1}\left(x_{1}+x_{3}+x_{4}\right)\left(2 x_{1}+x_{2}+x_{3}-x_{5}\right) \\
& \left(x_{2}+x_{3}+x_{4}+x_{5}\right)\left(x_{1}-x_{5}\right)\left(x_{1}-x_{4}-x_{5}\right) x_{4} .
\end{aligned}
$$

One can check that $\mathscr{D}$ is still accurate: Only the restriction to $H=$ $\operatorname{ker}\left(x_{4}\right)$ has exponents $(1,5,5,5)$ and only the restrictions to

$$
\begin{aligned}
& X_{1}=\operatorname{ker}\left(2 x_{1}+x_{2}+x_{3}\right) \cap \operatorname{ker}\left(2 x_{1}+x_{2}+2 x_{3}+x_{4}-x_{5}\right), \\
& X_{2}=\operatorname{ker}\left(x_{2}+x_{5}\right) \cap \operatorname{ker}\left(x_{2}+x_{3}+x_{4}\right)
\end{aligned}
$$

are free with exponents $\exp \left(\mathscr{D}^{X_{1}}\right)=\exp \left(\mathscr{D}^{X_{2}}\right)=(1,5,5)$. However, neither of those flats is contained in $H=\operatorname{ker}\left(x_{4}\right)$. Note further that $Y_{1}=X_{1} \cap H$ and also $Y_{2}=X_{2} \cap H$ are rank 3 flats with $\exp \left(\mathscr{D}^{Y_{1}}\right)=$ 
$\exp \left(\mathscr{D}^{Y_{2}}\right)=(1,5)$. In particular, the lack of a suitable rank 2 flat lying between $Y_{1}$ (or $Y_{2}$ ) and $H$ prevents $\mathscr{D}$ from being divisionally free.

It is also easily seen that the free but non-divisionally free rank 7 arrangement $\mathscr{B}$ constructed in [HR19] as a certain subarrangement of the Weyl arrangement of type $E_{7}$ is also still accurate.

In [CM20, §6], Cuntz and Mücksch checked that both $\mathscr{D}$ and $\mathscr{B}$ fail to be MAT-free.

Remark 5.6. In the recent paper [ATT20], Abe, Terao and Tran derive the freeness of all restrictions $\mathscr{A}(W)^{X}$ of Weyl arrangements $\mathscr{A}(W)$ to intersections $X$ where $\mathscr{A}(W)_{X} / X \cong \mathscr{A}\left(A_{1}^{2}\right)$. [ATT20, Thm. 1.5] asserts that the exponents of such a restriction $\mathscr{A}(W)^{X}$ are either given by removing the two highest exponents of $\mathscr{A}(W)$ or by removing the highest exponent and the middle exponent $h / 2$, where $h$ is the Coxeter number of $W$. Consequently, together with Corollary 1.4, their result implies the divisional freeness and hence also the almost accuracy of Weyl arrangements of rank at most 4.

5.2. Graphic arrangements. In this section we examine accuracy among free graphic arrangements. For basics on the latter, we refer to [OT92, Sec. 2.4].

Our next example shows that also free graphic arrangements which are always supersolvable and come from chordal graphs (cf. [ER94, Sec. 3]) may fail to be accurate in general.

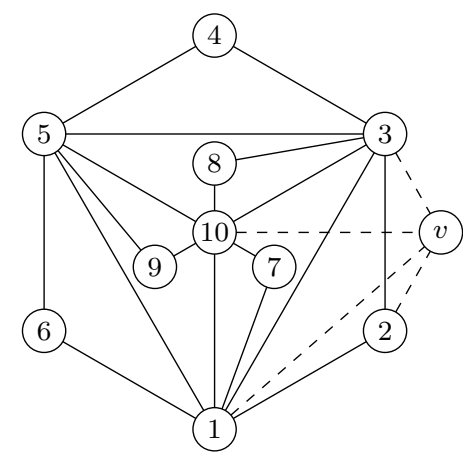

FiguRE 3. A chordal graph $\mathcal{G}$ giving rise to a graphic arrangement which is free, but not accurate, and an extension $\mathcal{G}^{\prime}$ by one vertex $v$ resulting in a graphic arrangement which is accurate.

Example 5.7. Let $\mathcal{G}=(\mathcal{V}, \mathcal{E})$ be the graph with vertex set $\mathcal{V}=$ $\{1, \ldots, 10\}$ and 18 edges $\mathcal{E}$, as shown in Figure 3 . It is easily seen that $(2,4,6,7,8,9,1,3,5,10)$ is a vertex elimination order for $\mathcal{G}$ (cf. [ER94, 
Sec. 3]). Then by [ER94, Thm. 3.3], the graphic arrangement $\mathscr{A}=$ $\mathscr{A}(\mathcal{G})=\left\{\operatorname{ker}\left(x_{i}-x_{j}\right) \mid(i, j) \in \mathcal{E}\right\}$ is free with exponents $\exp (\mathscr{A})=$ $(0,1,2, \ldots, 2,3) \in \mathbb{Z}^{10}$. From Figure 3 it is evident that up to symmetry there are only two different kinds of hyperplanes in $\mathscr{A}$. The hyperplane $H=\operatorname{ker}\left(x_{1}-x_{2}\right)$ is a representative of the first type and the hyperplane $K=\operatorname{ker}\left(x_{1}-x_{3}\right)$ is a representative of the second type. We easily see that

$$
\begin{aligned}
& \exp \left(\mathscr{A}^{H}\right)=(0,1,2, \ldots, 2,3) \in \mathbb{Z}^{9}, \\
& \exp \left(\mathscr{A}^{K}\right)=(0,1,1,2, \ldots, 2) \in \mathbb{Z}^{9} .
\end{aligned}
$$

Consequently, the graphic arrangement $\mathscr{A}$ is not accurate, in particular, it is not MAT-free, by Theorem 1.2. Of course, $\mathscr{A}$ is still almost accurate since it is supersolvable.

Further, from $\mathcal{G}$ we can construct a new graph, illustrating the behavior of accuracy with respect to restrictions and localizations, as follows. Extending $\mathcal{G}$ by one additional vertex $v$ and the edges indicated by the dashed lines in Figure 3 yields a new chordal graph $\mathcal{G}^{\prime}$. The corresponding graphic arrangement $\mathscr{B}:=\mathscr{A}\left(\mathcal{G}^{\prime}\right)$ has exponents $\exp (\mathscr{B})=(0,1,2, \ldots, 2,3,3,3) \in \mathbb{Z}^{11}$. It is not hard to see, by contracting the appropriate edges, that $\mathscr{B}$ is accurate. But for $H^{\prime}=\operatorname{ker}\left(x_{1}-x_{v}\right) \in \mathscr{B}$ (the hyperplane corresponding to the new edge $(1, v)$ of $\left.\mathcal{G}^{\prime}\right)$, we have $\mathscr{B}^{H^{\prime}}=\mathscr{A}$. Moreover, for

$$
X=\bigcap_{1 \leq i<j \leq 10} \operatorname{ker}\left(x_{i}-x_{j}\right)
$$

we also obtain $\mathscr{B}_{X} \cong \mathscr{A}$. This shows that in general, accuracy is neither inherited by restrictions (even if they are supersolvable), nor by localizations.

Lastly, one can show that every free graphic arrangement with fewer than 18 edges is still accurate. Thus our chordal arrangement $\mathscr{A}$ is the smallest one that fails to be accurate.

\subsection{Complex reflection arrangements and their restrictions.}

Let $G \subseteq \mathrm{GL}(V)$ be a finite, complex reflection group acting on the complex vector space $V=\mathbb{C}^{\ell}$. The reflection arrangement of $G$ in $V$ is the hyperplane arrangement $\mathscr{A}(G)$ consisting of the reflecting hyperplanes of the elements in $G$ acting as reflections on $V$.

Terao [Ter80b] has shown that every reflection arrangement $\mathscr{A}(G)$ is free and that the exponents of $\mathscr{A}(G)$ coincide with the coexponents of $G$, cf. [OT92, Prop. 6.59 and Thm. 6.60].

In view of Theorem 1.6, it is natural to examine accuracy for the larger class of complex reflection arrangements. Thanks to Remark 
1.7(v) and [Rö18, Prop. 2.12], accuracy and divisional freeness are compatible with products.

Theorem 5.8. Let $G$ be a complex reflection group with reflection arrangement $\mathscr{A}=\mathscr{A}(G)$. Then $\mathscr{A}$ is accurate if and only if it is divisionally free. This is the case if and only if $G$ has no irreducible factor isomorphic to one of the monomial groups $G(r, r, \ell), r>2, \ell>2$, or $G_{24}, G_{27}, G_{29}, G_{33}, G_{34}$.

Proof. The result follows readily from Definition 1.1, the classification of the divisionally free reflection arrangements from [Abe16, Cor. 4.7], and the exponents of the complex reflection arrangements and their restrictions, e.g. see [OT92, §6.4, App. C].

In view of Theorem 5.8 and the fact that all restrictions of complex reflection arrangements are free (thanks to [OT92, §6.4, App. D], [OT93], and [HR13]), it is natural to investigate accuracy among restrictions of complex reflection arrangements, not all of which are reflection arrangements again.

In order to derive our results, we require some further notation. Orlik and Solomon defined intermediate arrangements $\mathscr{A}_{\ell}^{k}(r)$ in [OS83, $\S 2]$ (cf. [OT92, §6.4]) which interpolate between the reflection arrangements of the monomial groups $G(r, r, \ell)$ and $G(r, 1, \ell)$. They show up as restrictions of the reflection arrangement of $G\left(r, r, \ell^{\prime}\right)$, for some $\ell^{\prime}$, [OS83, Prop. 2.14] (cf. [OT92, Prop. 6.84]).

For $\ell, r \geq 2$ and $0 \leq k \leq \ell$ the defining polynomial of $\mathscr{A}_{\ell}^{k}(r)$ is given by

$$
Q\left(\mathscr{A}_{\ell}^{k}(r)\right)=x_{1} \cdots x_{k} \prod_{\substack{1 \leq i<j \leq \ell \\ 0 \leq n<r}}\left(x_{i}-\zeta^{n} x_{j}\right),
$$

where $\zeta$ is a primitive $r$-th root of unity, so that $\mathscr{A}_{\ell}^{\ell}(r)=\mathscr{A}(G(r, 1, \ell))$ and $\mathscr{A}_{\ell}^{0}(r)=\mathscr{A}(G(r, r, \ell))$. For $k \neq 0, \ell$, these are not reflection arrangements themselves.

Next we recall [OS83, Props. 2.11, 2.13] (cf. [OT92, Props. 6.82, $6.85])$.

Proposition 5.9. Let $\mathscr{A}=\mathscr{A}_{\ell}^{k}(r)$ for $\ell, r \geq 2$ and $0 \leq k \leq \ell$. Then

(i) $\mathscr{A}$ is free with

$$
\exp \mathscr{A}=(1, r+1, \ldots,(\ell-2) r+1,(\ell-1) r-\ell+k+1),
$$

(ii) for $H \in \mathscr{A}$, the type of $\mathscr{A}^{H}$ is given in Table 1.

Lemma 5.10. Let $\mathscr{A}=\mathscr{A}_{\ell}^{k}(r)$ for $\ell, r \geq 2$ and $1 \leq k \leq \ell-1$. Then (i) for $r=2, \mathscr{A}$ is accurate; 


\begin{tabular}{|c|c|c|c|}
\hline$k$ & $\alpha_{H}$ & & Type of $\mathscr{A}^{H}$ \\
\hline 0 & arbitrary & & $\mathscr{A}_{\ell-1}^{1}(r)$ \\
\hline $1, \ldots, \ell-1$ & $x_{i}-\zeta x_{j}$ & $1 \leq i<j \leq k<\ell$ & $\mathscr{A}_{\ell-1}^{k-1}(r)$ \\
\hline $1, \ldots, \ell-1$ & $x_{i}-\zeta x_{j}$ & $1 \leq i \leq k<j \leq \ell$ & $\mathscr{A}_{\ell-1}^{k}(r)$ \\
\hline $1, \ldots, \ell-1$ & $x_{i}-\zeta x_{j}$ & $1 \leq k<i<j \leq \ell$ & $\mathscr{A}_{\ell-1}^{k+1}(r)$ \\
\hline $1, \ldots, \ell-1$ & $x_{i}$ & $1 \leq i \leq \ell$ & $\mathscr{A}_{\ell-1}^{\ell-1}(r)$ \\
\hline$\ell$ & arbitrary & & $\mathscr{A}_{\ell-1}^{\ell-1}(r)$ \\
\hline
\end{tabular}

TABLE 1. Restriction types of $\mathscr{A}_{\ell}^{k}(r)$

(ii) for $r>2, \mathscr{A}$ is accurate if and only if $r+k \geq \ell$.

Proof. Thanks to Proposition 5.9(ii), the restriction of an intermediate arrangement is again of this kind. So we only need to consider the restriction $\mathscr{A}^{H}$ to a hyperplane. It follows from Proposition 5.9 that firstly, if $r+k \geq \ell$, then for $H=\operatorname{ker} x_{i}$, the exponents of $\mathscr{A}^{H}$ are all but the largest one of $\mathscr{A}$. Secondly, if $r>2$ and $r+k<\ell$, then there is no restriction $\mathscr{A}^{H}$ with this property. So $\mathscr{A}$ is not accurate in this instance.

Finally, for $r=2$ and $1 \leq k<\ell-2$, if we take $H$ to be as in the fourth row of Table 1, then once again the exponents of $\mathscr{A}^{H}$ are all but the largest one of $\mathscr{A}$.

Thanks to Lemma 5.10, the following is the smallest example of a non-accurate member among the intermediate arrangements $\mathscr{A}_{\ell}^{k}(r)$.

Example 5.11. By Proposition 5.9, $\exp \left(\mathscr{A}_{5}^{1}(3)\right)=(1,4,7,9,10)$, and the possible exponents of restrictions are $(1,4,7,7),(1,4,7,8)$, and $(1,4,7,10)$. In particular, there is no hyperplane in $\mathscr{A}_{5}^{1}(3)$ whose restriction has got exponents $(1,4,7,9)$. Consequently, $\mathscr{A}_{5}^{1}(3)$ is not accurate.

Again thanks to Remark 1.7(v) and [Rö18, Prop. 2.12], accuracy and divisional freeness are compatible with products. So we can reduce to the case of irreducible $G$ when considering restrictions of reflection arrangements.

Theorem 5.12. Let $G$ be an irreducible complex reflection group with reflection arrangement $\mathscr{A}(G)$. Let $\mathscr{A}=\mathscr{A}(G)^{Y}$, for $Y \in L(\mathscr{A}) \backslash\{V\}$. Then $\mathscr{A}$ is accurate if and only if one of the following holds: 
(i) $G \neq G(r, r, \ell)$;

(ii) $G=G(r, r, \ell)$ and either $r=2$ or else $\mathscr{A}=\mathscr{A}_{\ell}^{k}(r)$ with $r+k \geq \ell$ for $r>2$.

Proof. This follows readily from Definition 1.1, Lemma 5.10, and the exponents of the restrictions of the irreducible reflection arrangements, e.g. see $[\mathrm{OT} 92, \S 6.4$, App. C].

Remark 5.13. It follows from Theorem 5.12 that in contrast to the case of complex reflection arrangements from Theorem 5.8, there are divisionally free restrictions of the latter that are not accurate. For, thanks to [Abe16, Thm. 5.6], all $\mathscr{A}_{\ell}^{k}(r)$ are divisionally free for $\ell, r \geq 3$ and $k \geq 1$.

Nevertheless, from the classification of the divisionally free restrictions of reflection arrangements from [Rö18, Thm. 3.3] and the exponents of the restrictions of the reflection arrangements, e.g. see [OT92, $\S 6.4$, App. C], it follows from Theorem 5.12 that if $G \neq G(r, r, \ell)$, then $\mathscr{A}(G)^{Y}$ is accurate if and only if it is divisionally free.

Note that $G(2,2, \ell)$ is the Coxeter group of type $D_{\ell}$ and thus the arrangements $\mathscr{A}_{\ell}^{k}(2)$ are restrictions of Coxeter arrangements of type $D$. As a consequence of Lemma 5.10, all such are accurate.

The following consequence of Theorem 5.12 shows that accuracy for Coxeter arrangements (Theorem 1.6) extends to their restrictions.

Corollary 5.14. Restrictions of Coxeter arrangements are accurate.

Observe that accuracy of Coxeter arrangements, Theorem 1.6, is a consequence of Theorem 1.2 and the fact that all such are MATfree, [CM20]. In contrast, restrictions of Coxeter arrangements are not MAT-free in general, see [CM20, Ex. 22]. So Corollary 5.14 does not follow from Theorem 1.2 and is independent from Theorem 1.6.

Finally, we present an example among the intermediate arrangements which shows that accuracy is not compatible with taking localizations.

Example 5.15. Let $\mathscr{A}=\mathscr{A}_{\ell}^{1}(r)$ for $\ell \geq 4$ and $r \geq \ell-1$. Let

$$
X=\bigcap_{\substack{2 \leq i<j \leq \ell \\ 0 \leq n<r}} \operatorname{ker}\left(x_{i}-\zeta^{n} x_{j}\right) \text {, }
$$

where $\zeta$ is a primitive $r$-th root of unity. Then $\mathscr{A}_{X} \cong \mathscr{A}_{\ell-1}^{0}(r)$. By Theorems 5.8 and $5.12, \mathscr{A}$ is accurate but $\mathscr{A}_{X} \cong \mathscr{A}(G(r, r, \ell-1))$ is not. 


\section{REFERENCES}

[Abe16] T. Abe, Divisionally free arrangements of hyperplanes, Invent. Math. 204(1), (2016), 317-346. 23, 24, 27, 29

$\left[\mathrm{ABC}^{+} 16\right]$ T. Abe, M. Barakat, M. Cuntz, T. Hoge, and H. Terao, The freeness of ideal subarrangements of Weyl arrangements, J. Eur. Math. Soc. (JEMS) 18 (2016), no. 6, 1339-1348. 3, 4, 5, 10, 12, 18

[AT16] T. Abe and H. Terao, Free filtrations of affine Weyl arrangements and the ideal-Shi arrangements, J. Algebraic Combin. 43 (2016), 33-44. 5, 20,21

[AT19] _ Multiple addition, deletion and restriction theorems for hyperplane arrangements, Proc. Amer. Math. Soc. 147 (2019), no. 11, 48354845. 5, 22

[ATT20] T. Abe, H. Terao, and T. N. Tran, On $A_{1}^{2}$ restrictions of Weyl arrangements, J. Algebraic Combin. (2020). https://doi.org/10.1007/s10801-020-00979-8 25

[AMR18] N. Amend, T. Möller, and G. Röhrle, Restrictions of aspherical arrangements. Topology Appl. 249 (2018), 67-72. 5

[Arn76] V. I. Arnold, Wave front evolution and equivariant Morse lemma, Comm. Pure Appl. Math. 29 (1976), 557-582. 2

[Arn79] _ Indices of singular points of I-forms on a manifold with boundary, convolution of invariants of reflection groups, and singular projections of smooth surfaces, Russian Math. Surveys 34 (1979), 1-42. 2

[Ath96] C. A. Athanasiadis, Characteristic polynomials of subspace arrangements and finite fields, Adv. Math. 122 (1996), no. 2, 193-233. 20

[Bou68] N. Bourbaki, Éléments de mathématique. Groupes et algèbres de Lie. Chapitre IV-VI, Actualités Scientifiques et Industrielles, No. 1337, Hermann, Paris, 1968. 4, 6, 18

[CM19] M. Cuntz and P. Mücksch, Supersolvable simplicial arrangements, Adv. in Appl. Math. 107 (2019), 32-73. 24

[CM20] _ MAT-free reflection arrangements, Electronic J. Comb. 27(1) (2020), \#P1.28. 3, 4, 5, 11, 24, 25, 29

[CRS19] M. Cuntz, G. Röhrle, and A. Schauenburg, Arrangements of ideal type are inductively free, Internat. J. Algebra Comput. 29 (2019), no. 5, 761773. 6

[Dou99] J. M. Douglass, The adjoint representation of a reductive group and hyperplane arrangements, Represent. Theory 3 (1999), 444-456. 3

[ER94] P. H. Edelman and V. Reiner, Free hyperplane arrangements between $A_{n-1}$ and $B_{n}$, Math. Z. 215 (1994), no. 3, 347-365. 25, 26

[ER96] , Free arrangements and rhombic tilings, Discrete Comput. Geom. 15 (1996), 307-340 21

[Grü09] B. Grünbaum, A catalogue of simplicial arrangements in the real projective plane, Ars Math. Contemp. 2 (2009), no. 1, 1-25. 5, 24

[HR13] T. Hoge and G. Röhrle, Reflection arrangements are hereditarily free, Tôhoku Math. J. 65 (2013), no. 3, 313-319. 27

[HR19] , Some remarks on free arrangements, 2019, arXiv:1903.01438. 24,25 
[OS83] P. Orlik and L. Solomon, Coxeter arrangements, Singularities, Part 2 (Arcata, Calif., 1981), Proc. Sympos. Pure Math., vol. 40, Amer. Math. Soc., Providence, RI, 1983, pp. 269-291. 2, 3, 27

[OST87] P. Orlik, L. Solomon, and H. Terao, On Coxeter arrangements and the Coxeter number, Complex analytic singularities, Adv. Stud. Pure Math., vol. 8, North-Holland, Amsterdam, 1987, pp. 461-477. 2, 3, 4, 5

[OT92] P. Orlik and H. Terao, Arrangements of hyperplanes, Grundlehren der mathematischen Wissenschaften, Springer, 1992. 2, 3, 5, 6, 7, 8, 9, 16, $21,23,24,25,26,27,29$

[OT93] Coxeter arrangements are hereditarily free, Tohoku Math. J. (2) 45 (1993), no. 3, 369-383. 3, 27

[Rö18] G. Röhrle, Divisionally free restrictions of reflection arrangements. Sém. Lothar. Combin. 77 ([2016-2018]), Art. B77e, 8 pp. 27, 28, 29

[Sai75] K. Saito, On the uniformization of complements of discriminant loci, in: Conference Notes, Amer. Math. Soc. Summer Institute, Williamstown, 1975. 2

[Sai80] Theory of logarithmic differential forms and logarithmic vector fields, J. Fac. Sci. Univ. Tokyo Sect. IA Math. 27 (1980), 265-291. 2

[Ter80a] H. Terao, Arrangements of hyperplanes and their freeness I, J. Fac. Sci. Univ. Tokyo 27 (1980), 293-320. 3, 9, 23

[Ter80b] - Free arrangements of hyperplanes and unitary reflection groups, Proc. Japan Acad. Ser. A Math. Sci. 56 (1980), no. 8, 389-392. 26

[Ter81]_, Generalized exponents of a free arrangement of hyperplanes and Shepherd-Todd-Brieskorn formula, Invent. Math. 63 no. 1 (1981) 159179. 2, 18, 19

[Yos04] M. Yoshinaga, Characterization of a free arrangement and conjecture of Edelman and Reiner, Invent. Math. 157 no. 2 (2004) 449-454. 21

Paul Mücksch, Fakultät für Mathematik, Ruhr-Universität Bochum, D-44780 Bochum, Germany

Email address: paul.muecksch@rub.de

Gerhard Röhrle, Fakultät für Mathematik, Ruhr-Universität Bochum, D-44780 Bochum, Germany

Email address: gerhard.roehrle@rub.de 